\title{
Theory and applications of supercycled symmetry-based recoupling sequences in solid-state nuclear magnetic resonance
}

\author{
Per Eugen Kristiansen \\ Department of Molecular Biosciences, University of Oslo, P.O. Box 1041-Blindern, 0316 Oslo, Norway \\ Marina Carravetta, Jacco D. van Beek, ${ }^{\text {a) }}$ Wai Cheu Lai, and Malcolm H. Levitt ${ }^{\text {b) }}$ \\ Chemistry Department, Southampton University, Southampton SO17 1BJ, United Kingdom
}

(Received 9 March 2006; accepted 24 April 2006; published online 20 June 2006)

\begin{abstract}
We present the theoretical principles of supercycled symmetry-based recoupling sequences in solid-state magic-angle-spinning NMR. We discuss the construction procedure of the SR26 pulse sequence, which is a particularly robust sequence for double-quantum homonuclear dipole-dipole recoupling. The supercycle removes destructive higher-order average Hamiltonian terms and renders the sequence robust over long time intervals. We demonstrate applications of the SR26 sequence to double-quantum spectroscopy, homonuclear spin counting, and determination of the relative orientations of chemical shift anisotropy tensors. (c) 2006 American Institute of Physics. [DOI: $10.1063 / 1.2205857]$
\end{abstract}

\section{INTRODUCTION}

Solid-state nuclear magnetic resonance (NMR) is an increasingly powerful method for addressing biomolecular and material structural problems. ${ }^{1-5}$ Many of the applications of solid-state NMR to molecular structure determination use radio-frequency pulse sequences which are sensitive to the through-space dipole-dipole coupling between nuclei. The presence of a finite dipole-dipole coupling indicates the spatial proximity of nuclei, while an accurate estimate of the magnitude of the coupling allows a determination of the internuclear distance. For example, picometer-resolution molecular structural information has been obtained on the chromophore of the membrane protein rhodopsin. ${ }^{6}$

For most macromolecular applications of solid-state NMR, the employed techniques must be compatible with magic-angle spinning (MAS), which provides good sensitivity and resolution. ${ }^{1-5}$ A wide variety of dipolar recoupling methods exists for the exploitation of dipole-dipole couplings in the presence of magic-angle spinning. ${ }^{7-26}$ These methods allow the determination of qualitative internuclear proximities and, in some cases, quantitative internuclear distances. ${ }^{7-14,18-23}$ In addition, the recoupling of nuclei under magic-angle-spinning conditions allows the excitation of multiple-quantum coherences, enabling the estimation of molecular torsional angles. ${ }^{12,27-35}$ The excitation of highorder multiple-quantum coherences in clusters of coupled spin-1/2 allows an estimate to be made of the number of nuclei in the cluster. ${ }^{32,36-42}$ The latter method, called "multiple-quantum spin counting," was initially demonstrated on static solids but has also been employed in magicangle-spinning NMR. ${ }^{40-42}$

The symmetry-based approach to pulse sequence design ${ }^{19-26}$ leads to a range of recoupling solutions appli-

\footnotetext{
a) Present address: ETH Zürich, Laboratorium für Physikalische Chemie, ETH-Hönggerberg, CH-8093 Zürich, Switzerland.

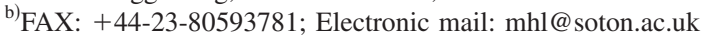

cable in a range of circumstances and allows the prediction of important features of the recoupling sequence, such as its orientation dependence and decoupling properties, on the basis of simple integer inequalities. Many of the symmetrybased recoupling sequences display a property called " $\gamma$ encoding." "18,26 This implies that the recoupled effective spin Hamiltonian is phase modulated by the Euler angle $\gamma_{M R}$, which is one of the three angles defining the orientation of the molecules with respect to the sample holder. Sequences displaying $\gamma$ encoding, such as $\mathrm{C} 7{ }^{19}$ POST-C $7,{ }^{20}$ and $\mathrm{R} 14_{2}^{6},{ }^{22}$ can achieve particularly high double-quantum efficiencies in unoriented samples. However, $\gamma$-encoded double-quantum recoupling sequences have not proven so suitable for longerrange excitation of multiple-quantum coherence, although attempts have been made. ${ }^{23}$ The main problem is their general lack of robustness at long recoupling times, where they are susceptible to interference from chemical shift anisotropies and radio-frequency phase errors. ${ }^{23,43}$

The robustness of symmetry-based sequences may be greatly improved by supercycling, i.e., repetition of the pulse sequence with an additional overall phase shift and/or a change in sign of all phases. We demonstrated recently a supercycled pulse sequence called SR $26,{ }^{25}$ which achieves relatively efficient homonuclear dipolar recoupling even for small dipolar interactions and relatively large chemical shift anisotropies. The type of supercycle employed for this sequence sacrifices the favorable features of $\gamma$ encoding for the sake of greater robustness. When applied in the context of small dipolar couplings, SR26 appears to have a better performance than other methods, including the highly successful DRAWS sequence, ${ }^{12-14}$ which has already been applied to many biomolecular problems. ${ }^{14,44}$ The SR26 sequence has been used successfully for three-dimensional structural studies of a zeolite framework. ${ }^{45,46}$

The SR26 sequence may be written explicitly as follows: 


$$
\begin{aligned}
& {\left[90_{76.15} 270_{256.15} 90_{283.85} 270_{103.85}\right]^{13}} \\
& {\left[90_{283.85} 270_{103.85} 90_{76.15} 270_{256.15}\right]^{13}} \\
& -\left[90_{103.85} 270_{283.85} 90_{256.15} 270_{76.15}\right]^{13} \\
& {\left[90_{256.15} 270_{76.15} 90_{103.85} 270_{283.85}\right]^{13},}
\end{aligned}
$$

where each pulse is denoted $\beta_{\phi}$ where $\beta$ is the flip angle and $\phi$ is the phase, both given in degrees. The superscripts denote 13 repetitions of the bracketed elements. The rf field amplitude is set so that the entire SR26 sequence has a duration of exactly 16 rotational periods. This requires a $\mathrm{rf}$ nutation frequency of 6.5 times the magic-angle-spinning frequency.

In this article we show that the operation of the SR26 supercycle may be understood by using average Hamiltonian theory, ${ }^{2,47,48}$ supported by numerical evaluations of the effective Hamiltonian. We also show a variety of experimental applications of SR26 which exploit its ability to perform homonuclear dipolar recoupling over relatively long distances. We use SR26 to determine accurate ${ }^{13} \mathrm{C}-{ }^{13} \mathrm{C}$ distances in selectively labeled material over a distance of around $0.4 \mathrm{~nm}$, we exploit double-quantum coherence generated by SR26 to determine the relative orientations of two chemical shift anisotropy (CSA) tensors, and we show that SR26 can be used to generate high-order intermolecular multiple-quantum coherences in homonuclear dipolarcoupled networks, in the presence of magic-angle spinning.

\section{THEORY}

\section{A. Symmetry-based recoupling}

The principles of symmetry-based recoupling sequences have been given in detail elsewhere. ${ }^{19-26} \mathrm{~A}$ brief review is given here in order to prepare the ground for the treatment of supercycles.

\section{Selection rule analysis}

There are two major classes of symmetry-based recoupling sequences, denoted $\mathrm{C} N_{n}^{\nu}$ and $\mathrm{R} N_{n}^{\nu}$. We will only deal with the $\mathrm{R} N_{n}^{\nu}$ class in this article. A pulse sequence with the symmetry $\mathrm{R} N_{n}^{\nu}$ is composed of $N$ elements $\left\{\mathcal{E}_{0} \mathcal{E}_{1} \cdots \mathcal{E}_{N-1}\right\}$, each of which has the same duration $\tau_{E}=n \tau_{r} / N$, where $\tau_{r}$ is a period of the magic-angle sample rotation, $\tau_{r}=\left|2 \pi / \omega_{r}\right|$, where the angular spinning frequency is $\omega_{r}$. Each element $\mathcal{E}_{q}$, with $q=0 \cdots N-1$, is derived from the same "basic element" $\mathcal{R}^{0}$, as follows:

$$
\mathcal{E}_{q}= \begin{cases}\mathcal{R}_{\pi \nu / N}^{0} & (q \text { even }) \\ \mathcal{R}_{-\pi \nu / N}^{0 \prime} & (q \text { odd }) .\end{cases}
$$

Here $\mathcal{R}^{0}$ is any sequence of resonant radio-frequency (rf) fields that rotates the resonant nuclei by an odd multiple of $\pi$ about the rotating-frame $x$ axis; the modified sequence $\mathcal{R}^{0 \prime}$ is derived from $\mathcal{R}^{0}$ by changing the sign of all rf phase shifts. In the case that $\mathcal{R}^{0}$ only involves phases that are multiples of $\pi$ (case of "amplitude modulation"), then $\mathcal{R}^{0 \prime}$ and $\mathcal{R}^{0}$ are identical. The subscripts in Eq. (2) refer to overall rf phase shifts. The complete $\mathrm{R} N_{n}^{\nu}$ sequence, denoted $\mathcal{S}_{0}$, therefore consists of $N / 2$ pairs of elements, with the two components

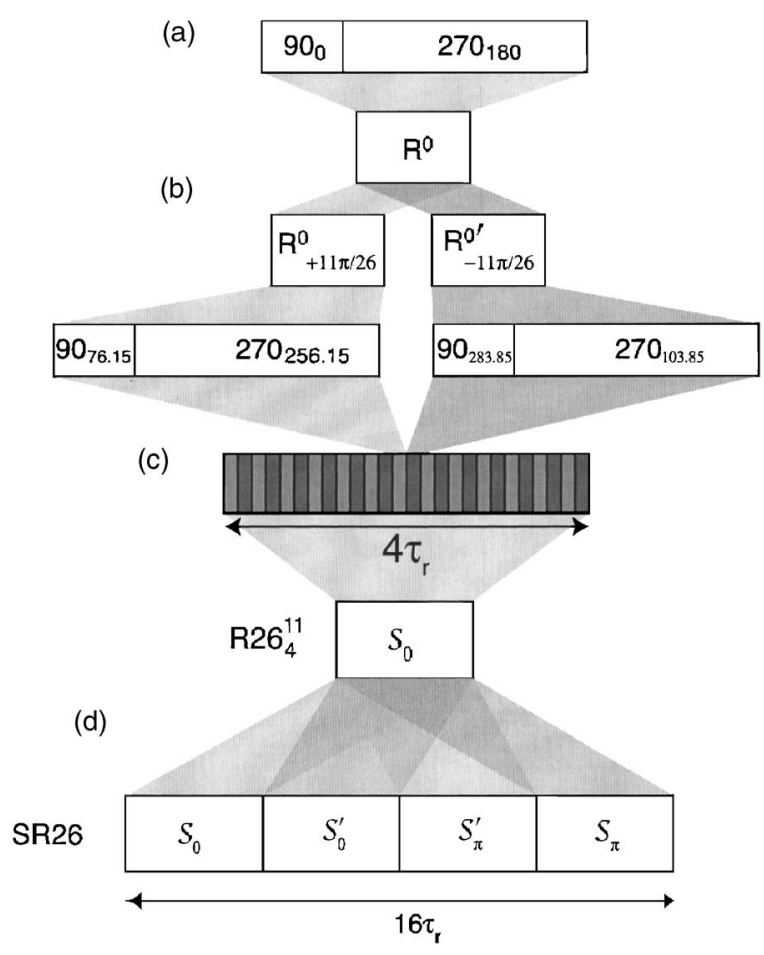

FIG. 1. Construction of the SR26 supercycle. (a) The $\mathcal{R}^{0}$ inversion element is a composite pulse of the form $90_{0} 270_{180}$. (b) Two elements are derived from $\mathcal{R}^{0}$ by (i) imposing an overall phase shift of $+\pi \nu / N$, leading to $\mathcal{R}_{+11 \pi / 26}^{0}$, or (ii) changing the sign of all phases, followed by an overall phase shift of $-\pi \nu / N$, leading to $\mathcal{R}_{-11 \pi / 26}^{0,}$ In the case of SR26, the symmetry numbers are $\nu=11$ and $N=26$, and $\mathcal{R}^{0 \prime}=\mathcal{R}^{0}$. (c) The R266 cycle $\mathcal{S}_{0}$ is constructed by concatenating $13 \mathcal{R}_{+11 \pi / 26}^{0} \mathcal{R}_{-11 \pi / 26}^{0 \prime}$ pairs. The total sequence duration is equal to four rotor periods. (d) The SR26 supercycle is constructed by concatenating $\mathcal{S}_{0}$ with the cycle $\mathcal{S}_{0}^{\prime}$ (derived by changing the sign of all phases), the cycle $\mathcal{S}_{\pi}$ (derived from $\mathcal{S}_{0}$ by an overall $\pi$ phase shift), and the cycle $\mathcal{S}_{\pi}^{\prime}$ (derived from $\mathcal{S}_{0}$ by an overall $\pi$ phase shift as well as a change in sign of all phases). The SR26 supercycle has a duration of 16 rotor periods.

of each pair having opposite overall phases $\pm \pi \nu / N$, i.e.,

$$
\mathcal{S}_{0}=\left[\mathcal{R}_{\pi \nu / N}^{0} \mathcal{R}_{-\pi \nu / N}^{0 \prime}\right]^{N / 2} \text {. }
$$

The SR26 sequence is built around a R26 ${ }_{4}^{11}$ cycle employing the basic element

$$
\mathcal{R}^{0}=\mathcal{R}^{0 \prime}=90_{0} 270_{180} .
$$

The explicit form of the $\mathrm{R} 26_{4}^{11}$ cycle is therefore

$$
\mathcal{S}_{0}=\left[90_{76.15} 270_{256.15} 90_{283.85} 270_{103.85}\right]^{13},
$$

as shown in Fig. 1.

The integrated propagator of the rf field between the time points $t_{a}$ and $t_{b}$ is denoted $U_{\mathrm{rf}}\left(t_{b}, t_{a}\right)$ and solves the equation

$$
\frac{d}{d t} U_{\mathrm{rf}}\left(t, t_{a}\right)=-i \mathcal{H}_{\mathrm{rf}}(t) U_{\mathrm{rf}}\left(t, t_{a}\right)
$$

The complete $\mathrm{R} N_{n}^{v}$ sequence lasts exactly $n$ rotor periods and will be denoted $\mathcal{S}_{0}$. Appendix A shows that the rf propagator for the $\mathrm{R} N_{n}^{\nu}$ sequence is given by 


$$
U_{\mathrm{rf}}\left(\mathcal{S}_{0}\right)=U_{\mathrm{rf}}\left(n \tau_{r}+t^{0}, t^{0}\right)=(-1)^{\nu+N / 2}
$$

where $t^{0}$ is the time point at which the sequence starts. The $\mathrm{R} N_{n}^{\nu}$ sequence may be regarded as a rf cycle, since the sign of the propagator is usually unimportant.

Average Hamiltonian theory ${ }^{2,47,48}$ conducted over the complete $\mathrm{R} N_{n}^{\nu}$ sequence leads to the following expression for the spin propagator:

$U\left(\mathcal{S}_{0}\right)=U_{\mathrm{rf}}\left(\mathcal{S}_{0}\right) \exp \left\{-i n r_{r} \overline{\mathcal{H}}\right\}=(-1)^{\nu+(1 / 2) N} \exp \left\{-i n \tau_{r} \overline{\mathcal{H}}\right\}$.

The effective Hamiltonian may be expressed as a convergent series (the Magnus expansion),

$$
\overline{\mathcal{H}}=\overline{\mathcal{H}}^{(1)}+\overline{\mathcal{H}}^{(2)}+\overline{\mathcal{H}}^{(3)}+\cdots,
$$

where each term is a superposition of many different components:

$$
\begin{aligned}
& \overline{\mathcal{H}}^{(1)}=\sum_{\mathbf{1}} \overline{\mathcal{H}}_{\mathbf{1}}^{(1)}, \\
& \overline{\mathcal{H}}^{(2)}=\sum_{\mathbf{2}} \sum_{\mathbf{1}} \overline{\mathcal{H}}_{\{\mathbf{2}, \mathbf{1}\}}^{(2)}, \\
& \overline{\mathcal{H}}^{(3)}=\sum_{\mathbf{3}} \sum_{\mathbf{2}} \sum_{\mathbf{1}} \overline{\mathcal{H}}_{\{\mathbf{3}, \mathbf{2}, \mathbf{1}\}}^{(3)} .
\end{aligned}
$$

For the sake of brevity, the bold index $\mathbf{1}$ is used to represent the set of quantum numbers $\left\{l_{1}, m_{1}, \lambda_{1}, \mu_{1}\right\}$ and similarly for 2 and 3. The sums are taken over all combinations of quantum numbers.

These quantum numbers have the following meaning: Each component of the interaction-frame spin Hamiltonian is classified according to its transformation properties under mechanical rotations of the molecular framework ("space" rotations), and under rotations of the nuclear magnetic moments ("spin" rotations). The space rotational properties are characterized by a rank $\ell$ and component $m$, which takes the values $m=\{-\ell,-\ell+1 \ldots \ell\}$. The spin rotational properties are characterized by a rank $\lambda$ and component $\mu$, which takes the values $\mu=\{-\lambda,-\lambda+1 \ldots \lambda\}$. The various nuclear spin interactions are distinguished by the values of $\ell$ and $\lambda$ : For homonuclear dipole-dipole interactions, $\{\ell, \lambda\}=\{2,2\}$; for isotropic chemical shifts, $\{\ell, \lambda\}=\{0,1\}$; and for chemical shift anisotropies, $\{\ell, \lambda\}=\{2,1\}$.

The average Hamiltonian theory of symmetry-based recoupling ${ }^{22-26}$ leads to the following selection rules for the first, second, and third-order terms:

$$
\overline{\mathcal{H}}_{\mathbf{1}}^{(1)}=0 \quad \text { if } m_{1} n-\mu_{1} \nu \neq \frac{1}{2} N Z_{\lambda_{1}}
$$

and

$$
\overline{\mathcal{H}}_{\{\mathbf{2}, \mathbf{1}\}}^{(2)}=0 \quad \text { if }\left\{\begin{array}{l}
m_{2} n-\mu_{2} \nu \neq \frac{1}{2} N Z_{\lambda_{2}} \wedge \\
m_{1} n-\mu_{1} \nu \neq \frac{1}{2} N Z_{\lambda_{1}} \wedge \\
\left(m_{2}+m_{1}\right) n-\left(\mu_{2}+\mu_{1}\right) \nu \neq \frac{1}{2} N Z_{\lambda_{2}+\lambda_{1}},
\end{array}\right.
$$

and

$$
\begin{aligned}
& \overline{\mathcal{H}}_{\{\mathbf{3 , 2 , 1 \}}}^{(3)}=0 \\
& \quad \text { if }\left\{\begin{array}{l}
m_{3} n-\mu_{3} \nu \neq \frac{1}{2} N Z_{\lambda_{3}} \wedge \\
m_{2} n-\mu_{2} \nu \neq \frac{1}{2} N Z_{\lambda_{2}} \wedge \\
m_{1} n-\mu_{2} \nu \neq \frac{1}{2} N Z_{\lambda_{1}} \wedge \\
\left(m_{3}+m_{2}\right) n-\left(\mu_{3}+\mu_{2}\right) \nu \neq \frac{1}{2} N Z_{\lambda_{3}+\lambda_{2}} \wedge \\
\left(m_{3}+m_{1}\right) n-\left(\mu_{3}+\mu_{1}\right) \nu \neq \frac{1}{2} N Z_{\lambda_{3}+\lambda_{1}} \wedge \\
\left(m_{2}+m_{1}\right) n-\left(\mu_{2}+\mu_{1}\right) \nu \neq \frac{1}{2} N Z_{\lambda_{2}+\lambda_{1}} \wedge \\
\left(m_{3}+m_{2}+m_{1}\right) n-\left(\mu_{3}+\mu_{2}+\mu_{1}\right) \nu \neq \frac{1}{2} N Z_{\lambda_{3}+\lambda_{2}+\lambda_{1}} .
\end{array}\right.
\end{aligned}
$$

The selection rules Eqs. (10) and (11) use the symbol $Z_{\lambda}$ to represent any integer (including zero) with the same parity as $\lambda$ : If $\lambda$ is even, then $Z_{\lambda} \in\{0, \pm 2, \pm 4 \cdots\}$; if $\lambda$ is odd, then $Z_{\lambda} \in\{ \pm 1, \pm 3 \cdots\}$. The symmetry-based approach to recoupling pulse sequence design allows one to select the desired recoupled interaction (to first order) through the simple integer inequality given in Eq. (10).

One of the most successful applications of symmetrybased recoupling has been in the design of $\gamma$-encoded double-quantum recoupling sequences. ${ }^{18-23}$ These sequences have the following properties: (i) all CSA terms are forbidden by the first-order selection rule Eq. (10); (ii) all homonuclear dipole-dipole (DD) coupling terms are forbidden by the first-order selection rule Eq. (10), except for some double-quantum terms $(\mu= \pm 2)$; and (iii) each doublequantum term is associated with a single symmetry-allowed spatial component $m$. A compilation of symmetries with these properties has been given; ${ }^{26}$ two examples are $\mathrm{R} 14_{2}^{6}$ (Ref. 23) and R26 ${ }_{4}^{11}$ (Ref. 25). For both of these sequences, the only symmetry-allowed first-order dipole-dipole coupling terms have the quantum numbers $\{\ell, m, \lambda, \mu\}=\{2,-1,2,2\}$ and $\{2,1,2,-2\}$. If the homonuclear $J$ coupling is ignored, the first-order average Hamiltonian for recoupling of isolated spin-1/2 pairs $I_{j}$ and $I_{k}$ is given by a pure double-quantum term

$$
\overline{\mathcal{H}}^{(1)}=\overline{\mathcal{H}}_{\mathrm{DQ}},
$$

where

$$
\overline{\mathcal{H}}_{\mathrm{DQ}}=\bar{\omega}_{2-122}^{j k} \frac{1}{2} I_{j}^{+} I_{k}^{+}+\bar{\omega}_{212-2}^{j k} \frac{1}{2} I_{j} I_{k} .
$$

Here the complex amplitude of a recoupled interaction $\Lambda$ is given in general by

$$
\bar{\omega}_{\ell m \lambda \mu}^{\Lambda}=\kappa_{\ell m \lambda \mu}\left[A_{\ell m}^{\Lambda}\right]^{R} \exp \left\{-i m\left(\alpha_{R L}^{0}-\omega_{r} t^{0}\right)\right\} .
$$

Here $\kappa_{\ell m \lambda \mu}$ is the first-order scaling factor, which depends on the quantum number of the recoupled term; the pulse sequence symmetry numbers $N, n$, and $\nu$; and the basic element $\mathcal{R}^{0}$. Explicit expressions for the scaling factor are given in Ref. 49. The term $\left[A_{\ell m}^{\Lambda}\right]^{R}$ represents the $m$ th component of the $\ell$ th-rank interaction $\Lambda$, expressed in the rotor-fixed frame $R$ ( $z$ axis along the sample rotation axis). The angle $\alpha_{R L}^{0}$ defines the orientation of the rotor at the start of the recoupling sequence (time point $t^{0}$ ). In the case of the dipoledipole interaction $(\ell=2, \Lambda=j k)$, the relevant rotor-frame interaction components are given by 


$$
\left[A_{2 m}^{j k}\right]^{R}=\sqrt{6} b_{j k} d_{0 m}^{2}\left(\beta_{P R}^{j k}\right) \exp \left\{-i m \gamma_{P R}^{j k}\right\},
$$

where $\left\{\beta_{P R}^{j k}, \gamma_{P R}^{j k}\right\}$ are two of the Euler angles relating the principal axis frame of the dipole-dipole interaction with the rotor-fixed frame. The dipole-dipole internuclear coupling constant is related to the inverse cube of the internuclear distance $r_{j k}$ through

$$
b_{j k}=-\frac{\mu_{0}}{4 \pi} \frac{\gamma^{2} \hbar}{r_{j k}^{3}}
$$

These equations show that only the phase of the complex amplitude $\bar{\omega}^{j k}$ depends on the Euler angle $\gamma_{P R}^{j k}$ : hence the term " $\gamma$ encoding." This property minimizes the orientation dependence of the recoupling, leading to relatively good efficiency in powder samples. For isolated pairs of spin-1/2, the first-order average Hamiltonian in Eq. (13) leads to a maximum efficiency of $73 \%$ for double-quantum filtering of powder NMR signals. ${ }^{19}$

\section{Chemical shift interference}

The first-order selection rule analysis predicts that symmetries such as $\mathrm{R} 14_{2}^{6}$ and $\mathrm{R} 26_{4}^{11}$ suppress the effects of chemical shifts. However, in practice, chemical shifts (both isotropic resonance offset effects and chemical shift anisotropies) can be several orders of magnitude larger than the dipole-dipole interactions. A first-order analysis is often insufficient, especially in the case of small dipole-dipole interactions. In the following analysis, we assume that the terms $\overline{\mathcal{H}}^{(2)}$ and $\overline{\mathcal{H}}^{(3)}$ are dominated by chemical shift interactions, including both isotropic chemical shifts $\{\ell, \lambda\}=\{0,1\}$ and chemical shift anisotropies $\{\ell, \lambda\}=\{2,1\}$.

All average Hamiltonian terms involving only chemical shifts involve commutators between single-spin first-rank interactions. Such terms must therefore be proportional to the angular momentum operators of the spins $I_{j}$ and $I_{k}$. The higher-order chemical shift interactions therefore give rise to average Hamiltonian contributions of the form

$$
\overline{\mathcal{H}}^{(2)}+\overline{\mathcal{H}}^{(3)} \cong \overline{\mathcal{H}}_{x}+\overline{\mathcal{H}}_{y}+\overline{\mathcal{H}}_{z},
$$

where

$$
\begin{aligned}
& \overline{\mathcal{H}}_{x}=\bar{\omega}_{j x} I_{j x}+\bar{\omega}_{k x} I_{k x}, \\
& \overline{\mathcal{H}}_{y}=\bar{\omega}_{j y} I_{j y}+\bar{\omega}_{k y} I_{k y}, \\
& \overline{\mathcal{H}}_{z}=\bar{\omega}_{j z} I_{j z}+\bar{\omega}_{k z} I_{k z} .
\end{aligned}
$$

The chemical shift Hamiltonian components $\overline{\mathcal{H}}_{x}, \overline{\mathcal{H}}_{y}$, and $\overline{\mathcal{H}}_{z}$ are, in general, strongly dependent on the chemical shift parameters and the molecular orientation.

Analysis of the second-order selection rule Eq. (11) shows that the symmetries $\mathrm{R} 14_{2}^{6}$ and $\mathrm{R} 26_{4}^{11}$ are inequivalent with respect to the second-order CSA terms: R14 ${ }_{2}^{6}$ has 20 symmetry-allowed CSA $\times \mathrm{CSA} \overline{\mathcal{H}}^{(2)}$ terms, while $\mathrm{R} 26_{4}^{11}$ has only 8 symmetry-allowed $\mathrm{CSA} \times \mathrm{CSA} \overline{\mathcal{H}}^{(2)}$ terms. This qualitative analysis therefore predicts that $\mathrm{R} 26_{4}^{11}$ is more robust with respect to CSA interference than $\mathrm{R} 14_{2}^{6}$. Further examination of the CSA $\times$ CSA $\overline{\mathcal{H}}^{(2)}$ terms in the case of $\mathrm{R}_{26}^{11}\left(N=26, n=4, \nu=11, \ell_{2}=\ell_{1}=2\right.$, and $\left.\lambda_{2}=\lambda_{1}=1\right)$ shows that the only symmetry-allowed terms have the form $\overline{\mathcal{H}}_{2, m, 1, \pm 1 ; 2,-m, 1, \mp 1}^{(2)}$, with $m= \pm 1, \pm 2$. The total Zeeman quantum number for these terms is therefore $\mu_{2}+\mu_{1}=0$. Similarly, the only second-order isotropic shift terms have the form $\overline{\mathcal{H}}_{0,0,1, \pm 1 ; 0,0,1, \mp 1}^{(2)}$, which also has $\mu_{2}+\mu_{1}=0$. Hence, in the case of R26 $6_{4}^{11}$, the second-order Hamiltonian $\overline{\mathcal{H}}^{(2)}$ only contributes to the longitudinal chemical shift term $\overline{\mathcal{H}}_{z}$.

The third-order Hamiltonian contributes to each of $\overline{\mathcal{H}}_{x}$, $\overline{\mathcal{H}}_{y}$, and $\overline{\mathcal{H}}_{z}$. A symmetry analysis based on Eq. (12) shows that there are 96 longitudinal symmetry-allowed third-order terms, as against 348 transverse symmetry-allowed thirdorder terms. The conclusion may be drawn that the longitudinal terms $\overline{\mathcal{H}}_{z}$ derives mainly from the second-order chemical shift term, while the transverse terms $\overline{\mathcal{H}}_{x}$ and $\overline{\mathcal{H}}_{y}$ are mainly due to the third-order chemical shift terms, in the case of $\mathrm{R} 26_{4}^{11}$. This property will be used later when analyzing the effect of supercycling.

The chemical-shift-dominated terms $\overline{\mathcal{H}}_{x}, \overline{\mathcal{H}}_{y}$, and $\overline{\mathcal{H}}_{z}$ do not commute with $\overline{\mathcal{H}}_{\mathrm{DQ}}$ and can be highly damaging for the double-quantum efficiency, especially in the case of small dipole-dipole interactions. The destructive effect of these terms has been documented. ${ }^{22,43}$

\section{Instrumental phase errors}

$\mathrm{R} N_{n}^{\nu}$ sequences suffer from a hypersensitivity to the radio-frequency phase shift. It has been demonstrated ${ }^{22}$ that rf phase shift errors of a fraction of a degree may have a strong detrimental influence on the double-quantum excitation efficiency, especially when recoupling is attempted over medium-range or long internuclear distances.

The reason for the phase-shift hypersensitivity is that the rf propagator $U_{\mathrm{rf}}\left(\mathcal{S}_{0}\right)$ is not proportional to the unity operator if the experimental phases are misset. Suppose that the pulse sequence elements are defined by

$$
\mathcal{E}_{q}= \begin{cases}\mathcal{R}_{\Phi}^{0}, & q \text { even } \\ \mathcal{R}_{-\Phi}^{0 \prime}, & q \text { odd },\end{cases}
$$

instead of by Eq. (2), where the phase shift is

$$
\Phi=\frac{\pi \nu}{N}+\epsilon,
$$

and $\epsilon$ is an instrumental error (or a deliberate fine phase adjustment). Appendix A shows that the overall propagator in this case is given by

$$
U_{\mathrm{rf}}\left(\mathcal{S}_{0}\right)=(-1)^{\nu+N / 2} \exp \left\{i 2 N \epsilon I_{z}\right\} .
$$

The sign of the propagator may be ignored for most purposes, but the second term shows that an error $\epsilon$ in the rf phase shift leads to a rotation of the spins around the $z$ axis on completion of each $\mathrm{R} N_{n}^{v}$ sequence. The rotation angle corresponds to the phase error magnified by the factor $-2 N$. In the case of $\mathrm{R} 26_{4}^{11}$, this multiplication factor is -52 , which means that a $0.1^{\circ}$ error in the size of the phase shift leads to an erroneous rotation of the nuclei by $-5^{\circ}$ after the comple- 
tion of just one $\mathrm{R} 26_{4}^{11}$ cycle. Since many recoupling cycles are often required, and this rotation accumulates between consecutive cycles, this feature places almost impossible demands on the phase shift accuracy. To make matters worse, the rf phase shift as experienced by the spins is influenced by other factors than the rf phase shift at the radio-frequency synthesis stage. For example, in the case of a sample rotating in an inhomogeneous rf field, the effective rf phase is modulated by the change in direction of the rf field experienced by each moving volume element. NMR phenomena associated with this effect have been demonstrated. ${ }^{10,50}$ Hence, the extreme phase-shift sensitivity of the $\mathrm{R} N_{n}^{\nu}$ sequences can probably not be addressed purely by improvements in the radiofrequency synthesis hardware.

\section{Effective Hamiltonian}

Equations (7), (13), (18), and (21) may be combined to give the following approximate expression for the spin propagator under a realistic symmetry-based doublequantum recoupling sequence:

$$
\begin{aligned}
U\left(\mathcal{S}_{0}\right) \cong & (-1)^{\nu+N / 2} \exp \left\{i 2 N \epsilon I_{z}\right\} \\
& \times \exp \left\{-i n \tau_{r}\left(\overline{\mathcal{H}}_{\mathrm{DQ}}+\overline{\mathcal{H}}_{z}+\overline{\mathcal{H}}_{x}+\overline{\mathcal{H}}_{y}\right)\right\} .
\end{aligned}
$$

This propagator may be interpreted as the result of a constant effective Hamiltonian, applied during the entire $n \tau_{r}$ interval,

$$
U\left(\mathcal{S}_{0}\right)=\exp \left\{-\operatorname{in} \tau_{r} \overline{\mathcal{H}}\left(\mathcal{S}_{0}\right)\right\}
$$

If the phase shift error $\epsilon$ is small, and the double-quantum and chemical shift terms $\overline{\mathcal{H}}_{\mathrm{DQ}}, \overline{\mathcal{H}}_{z}, \overline{\mathcal{H}}_{x}$, and $\overline{\mathcal{H}}_{y}$ are also small compared to the inverse of the cycle period $n \tau_{r}$, the Baker-Campbell-Hausdorff $(\mathrm{BCH})$ formula $^{2}$

$$
e^{B} e^{A}=\exp \left\{(B+A)+\frac{1}{2}[B, A]+\cdots\right\}
$$

may be used to obtain an approximate expression for the effective Hamiltonian over the complete $\mathrm{R} N_{n}^{\nu}$ sequence,

$$
\begin{aligned}
\overline{\mathcal{H}}\left(\mathcal{S}_{0}\right) \cong & \left(\overline{\mathcal{H}}_{\mathrm{DQ}}+\overline{\mathcal{H}}_{z}+\overline{\mathcal{H}}_{x}+\overline{\mathcal{H}}_{y}-\frac{2 N \epsilon}{n \tau_{r}} I_{z}\right) \\
& +i N \epsilon\left[\overline{\mathcal{H}}_{\mathrm{DQ}}+\overline{\mathcal{H}}_{x}+\overline{\mathcal{H}}_{y}, I_{z}\right] .
\end{aligned}
$$

The phase-shift error appears in the average Hamiltonian as an effective field along the $z$ axis, suggesting that a deliberate misset of the phase shift may be used to compensate the second-order chemical shift term, which contributes to $\overline{\mathcal{H}}_{z}$. A fine phase adjustment does indeed lead to improved performance in the case of large chemical shift interactions. ${ }^{22}$ However, this compensation method only leads to limited improvements in the performance of the pulse sequence, since the chemical shift interference terms are strongly orientation dependent.

The average Hamiltonian in Eq. (25) also displays a term due to the interference between the phase-shift error and the double-quantum and chemical shift Hamiltonians. In the discussion below, this term will be ignored.

\section{B. Modified cycles}

A supercycle is constructed by concatenating a set of modified rf cycles, which are usually the same duration as the original cycle but which differ in the radio-frequency phases or in the order of the component elements.

The operations which are used in the construction of the $\mathrm{SR}_{2} 6_{4}^{11}$ supercycle are as follows.

(1) Apply an overall phase shift of $\pi$ (generating a $\pi$-shifted cycle). If the original cycle is denoted $\mathcal{S}_{0}$, the phase-shifted cycle is denoted $\mathcal{S}_{\pi}$.

(2) Change the sign of all rf phase shifts (generating a phase-inverted cycle). If the original cycle is denoted $\mathcal{S}_{0}$, the phase-shifted cycle is denoted $\mathcal{S}_{0}^{\prime}$.

(3) A combination of the two (generating a $\pi$-shifted phase-inverted cycle). If the original cycle is denoted $\mathcal{S}_{0}$, the phase-shifted phase-inverted cycle is denoted $\mathcal{S}_{\pi}^{\prime}$

The effects of these transformations on the overall spin propagator are analyzed in Appendix B. The results are as follows.

\section{1. $\pi$-shifted cycles}

Appendix B-shows that a $\pi$ phase shift of all rf pulses rotates the effective Hamiltonian by $\pi$ about the $z$ axis. This inverts the sign of all odd-quantum terms, leaving all evenquantum terms unchanged. The effective Hamiltonians for a $\pi$-shifted cycle and non-phase-shifted cycle are related through

$$
\overline{\mathcal{H}}\left(\mathcal{S}_{\pi}\right) \cong \overline{\mathcal{H}}_{\mathrm{DQ}}\left(\mathcal{S}_{0}\right)+\overline{\mathcal{H}}_{z}\left(\mathcal{S}_{0}\right)-\overline{\mathcal{H}}_{x}\left(\mathcal{S}_{0}\right)-\overline{\mathcal{H}}_{y}\left(\mathcal{S}_{0}\right)-\frac{2 N \epsilon}{n \tau_{r}} I_{z} .
$$

\section{Phase-inverted cycles}

If the cycle $\mathcal{S}_{0}$ corresponds to a sequence with the symmetry $\mathrm{R} N_{n}^{\nu}$ based on the element $\mathcal{R}^{0}$, then the phase-inverted cycle $\mathcal{S}_{0}^{\prime}$ corresponds to a sequence with the symmetry $\mathrm{R} N_{n}^{-\nu}$, based on the phase-inverted element $\mathcal{R}^{0 \prime}$. If the basic element $\mathcal{R}^{0}$ only contains phase shifts that are multiples of $\pi$, then $\mathcal{R}^{0 \prime}$ is identical to $\mathcal{R}^{0}$.

As shown in AppendixB, the relationship between average Hamiltonian terms for cycles related by a phase inversion depends on the sum of the spin ranks for the participating interactions [see Eq. (97)]. For example, the doublequantum Hamiltonian $\overline{\mathcal{H}}_{\mathrm{DQ}}$ derives from the dipole-dipole interaction, which has spin rank 2. The relationship between this average Hamiltonian for the phase-inverted and nonphase-inverted cycles is given by

$$
\overline{\mathcal{H}}_{\mathrm{DQ}}\left(\mathcal{S}_{0}^{\prime}\right)=\Pi_{x} \overline{\mathcal{H}}_{\mathrm{DQ}}\left(\mathcal{S}_{0}\right) \Pi_{x}^{\dagger} .
$$

The relationships for the chemical shift terms are more complex. The term $\overline{\mathcal{H}}_{z}$ derives predominantly from the secondorder chemical shift cross terms, which have a spin rank sum of 2. As shown in Appendix B, phase inversion leads to a sign change for this term, 


$$
\overline{\mathcal{H}}_{z}\left(\mathcal{S}_{0}^{\prime}\right) \cong \Pi_{x} \overline{\mathcal{H}}_{z}\left(\mathcal{S}_{0}\right) \Pi_{x}^{\dagger}=-\overline{\mathcal{H}}_{z}\left(\mathcal{S}_{0}\right) .
$$

In the case of $\mathrm{R}_{2} 6_{4}^{11}$, the transverse interference terms $\overline{\mathcal{H}}_{x}$ and $\overline{\mathcal{H}}_{y}$ are derived predominantly from the third-order chemical shift terms, which have a rank sum of 3 . The phaseinversion relationships are as follows:

$$
\overline{\mathcal{H}}_{x}\left(\mathcal{S}_{0}^{\prime}\right) \cong-\Pi_{x} \overline{\mathcal{H}}_{x}\left(\mathcal{S}_{0}\right) \Pi_{x}^{\dagger}=-\overline{\mathcal{H}}_{x}\left(\mathcal{S}_{0}\right)
$$

and

$$
\overline{\mathcal{H}}_{y}\left(\mathcal{S}_{0}^{\prime}\right) \cong-\Pi_{x} \overline{\mathcal{H}}_{y}\left(\mathcal{S}_{0}\right) \Pi_{x}^{\dagger}=+\overline{\mathcal{H}}_{y}\left(\mathcal{S}_{0}\right) .
$$

The effective Hamiltonian for a phase-inverted cycle is therefore given approximately by

$$
\begin{aligned}
\overline{\mathcal{H}}\left(\mathcal{S}_{0}^{\prime}\right) \cong & \Pi_{x} \overline{\mathcal{H}}_{\mathrm{DQ}}\left(\mathcal{S}_{0}\right) \Pi_{x}^{\dagger}-\overline{\mathcal{H}}_{z}\left(\mathcal{S}_{0}\right)-\overline{\mathcal{H}}_{x}\left(\mathcal{S}_{0}\right) \\
& +\overline{\mathcal{H}}_{y}\left(\mathcal{S}_{0}\right)+\frac{2 N \epsilon}{n \tau_{r}} I_{z} .
\end{aligned}
$$

\section{3. $\pi$-shifted phase-inverted cycles}

If the rf phase is shifted by $\pi$ as well as being inverted, the previous operations are combined. The effective Hamiltonian is given by

$$
\begin{aligned}
\overline{\mathcal{H}}\left(\mathcal{S}_{\pi}^{\prime}\right) \cong & \Pi_{x} \overline{\mathcal{H}}_{\mathrm{DQ}}\left(\mathcal{S}_{0}\right) \Pi_{x}^{\dagger}-\overline{\mathcal{H}}_{z}\left(\mathcal{S}_{0}\right)+\overline{\mathcal{H}}_{x}\left(\mathcal{S}_{0}\right) \\
& -\overline{\mathcal{H}}_{y}\left(\mathcal{S}_{0}\right)+\frac{2 N \epsilon}{n \tau_{r}} I_{z} .
\end{aligned}
$$

\section{Supercycles}

The error terms in the average Hamiltonian may be reduced or eliminated by concatenating modified cycles. The stabilizing effect of supercycling on the average Hamiltonian has been examined in detail in the context of triple-quantum recoupling. ${ }^{48,51,52}$ Here we examine the effect of supercycling on symmetry-based recoupling double-quantum sequences.

The most relevant combinations of cycles are as follows.

\section{The supercycle $S_{0} S_{\pi}$}

If the original cycle $\mathcal{S}_{0}$ is followed by the phase-shifted cycle $\mathcal{S}_{\pi}$, the overall propagator is

$$
U\left(\mathcal{S}_{0} \mathcal{S}_{\pi}\right)=\exp \left\{-\operatorname{in} \tau_{r} \overline{\mathcal{H}}\left(\mathcal{S}_{\pi}\right)\right\} \exp \left\{-\operatorname{in} \tau_{r} \overline{\mathcal{H}}\left(\mathcal{S}_{0}\right)\right\},
$$

where the effective Hamiltonians are given by Eqs. (25) and (26). If the effective Hamiltonian terms are sufficiently small, the BCH formula [Eq. (24)] may be used to derive the effective Hamiltonian of the supercycle,

$$
\begin{aligned}
\overline{\mathcal{H}}\left(\mathcal{S}_{0} \mathcal{S}_{\pi}\right) \cong & \frac{1}{2}\left(\overline{\mathcal{H}}\left(\mathcal{S}_{\pi}\right)+\overline{\mathcal{H}}\left(\mathcal{S}_{0}\right)\right) \\
& +i \frac{1}{4} n \tau_{r}\left[\overline{\mathcal{H}}\left(\mathcal{S}_{\pi}\right), \overline{\mathcal{H}}\left(\mathcal{S}_{0}\right)\right] .
\end{aligned}
$$

The supercycle effective Hamiltonian is therefore given approximately by

$$
\overline{\mathcal{H}}\left(\mathcal{S}_{\pi} \mathcal{S}_{0}\right) \cong \overline{\mathcal{H}}_{\mathrm{DQ}}\left(\mathcal{S}_{0}\right)+\overline{\mathcal{H}}_{z}\left(\mathcal{S}_{0}\right)-\frac{2 N \epsilon}{n \tau_{r}} I_{z},
$$

which shows that the supercycle $\mathcal{S}_{0} \mathcal{S}_{\pi}$ eliminates the transverse chemical shift terms $\overline{\mathcal{H}}_{x}$ and $\overline{\mathcal{H}}_{y}$ to first order, but has no effect on the phase-shift sensitivity, or the dominant second-order chemical shift term $\overline{\mathcal{H}}_{z}$. The $\mathcal{S}_{0} \mathcal{S}_{\pi}$ supercycle is therefore of limited use.

\section{The supercycle $S_{0} S_{0}^{\prime}$}

The $\mathrm{BCH}$ formula may be used together with Eqs. (25) and (31) to derive the effective Hamiltonian of the $\mathcal{S}_{0} \mathcal{S}_{0}^{\prime}$ supercycle,

$$
\overline{\mathcal{H}}\left(\mathcal{S}_{0} \mathcal{S}_{0}^{\prime}\right) \cong \frac{1}{2}\left(\overline{\mathcal{H}}_{\mathrm{DQ}}\left(\mathcal{S}_{0}\right)+\Pi_{x} \overline{\mathcal{H}}_{\mathrm{DQ}}\left(\mathcal{S}_{0}\right) \Pi_{x}^{\dagger}\right)+\overline{\mathcal{H}}_{y}\left(\mathcal{S}_{0}\right) .
$$

The second-order BCH term has been neglected.

Equation (36) shows that the effective Hamiltonian for the $\mathcal{S}_{0} \mathcal{S}_{0}^{\prime}$ supercycle is free of the second-order $\overline{\mathcal{H}}_{z}$ chemical shift term and the phase error term, although the third-order chemical shift term $\overline{\mathcal{H}}_{y}$ is still present. However, the increased robustness with respect to chemical shifts and phase errors is accompanied by a modification of the doublequantum term. The effect of this modification is examined in more detail below.

\section{The supercycle $S_{0} S_{0}^{\prime} S_{\pi}^{\prime} S_{\pi}$}

The twofold supercycles discussed above may be combined to give the four-step supercycle $\mathcal{S}_{0} \mathcal{S}_{0}^{\prime} \mathcal{S}_{\pi}^{\prime} \mathcal{S}_{\pi}$. The BCH formula may be used together with Eqs. (25), (26), (31), and (32) to derive the approximate effective Hamiltonian of this supercycle,

$$
\overline{\mathcal{H}}\left(\mathcal{S}_{0} \mathcal{S}_{0}^{\prime} \mathcal{S}_{\pi}^{\prime} \mathcal{S}_{\pi}\right) \cong \frac{1}{2}\left(\overline{\mathcal{H}}_{\mathrm{DQ}}\left(\mathcal{S}_{0}\right)+\Pi_{x} \overline{\mathcal{H}}_{\mathrm{DQ}}\left(\mathcal{S}_{0}\right) \Pi_{x}^{\dagger}\right) .
$$

The four-step $\mathcal{S}_{0} \mathcal{S}_{0}^{\prime} \mathcal{S}_{\pi}^{\prime} \mathcal{S}_{\pi}$ supercycle therefore eliminates all chemical shift interference and phase error terms, to first order in the $\mathrm{BCH}$ expansions.

The SR26 supercycle given in Eq. (1) corresponds to a $\mathcal{S}_{0} \mathcal{S}_{0}^{\prime} \mathcal{S}_{\pi}^{\prime} \mathcal{S}_{\pi}$ expansion of the R26 ${ }_{4}^{11}$ cycle $\mathcal{S}_{0}$ in Eq. (4). The construction of SR26 is summarized in Fig. 1.

Equation (37) may be combined with Eq. (14) to obtain a more detailed result for the average Hamiltonian of SR26,

$$
\overline{\mathcal{H}}(S R 26) \cong \bar{\omega}_{\mathrm{SR} 26} I_{x}^{\mathrm{DQ}},
$$

where the amplitude of the recoupled double-quantum term is

$$
\bar{\omega}_{\mathrm{SR} 26}=\operatorname{Re}\left\{\bar{\omega}_{2-122}^{j k}\right\}
$$

and the double-quantum $x$ operator is

$$
I_{x}^{\mathrm{DQ}}=\frac{1}{2}\left(I_{j}^{+} I_{k}^{+}+I_{j}^{-} I_{k}\right) .
$$

The amplitude of the double-quantum term is given explicitly by 


$$
\begin{aligned}
\bar{\omega}_{\mathrm{SR} 26} & =\sqrt{6} b_{j k} d_{0-1}^{2}\left(\beta_{P R}^{j k}\right)\left|\kappa_{2-122}\right| \cos \left(\gamma_{P R}^{j k}+\xi\right) \\
& =-\frac{3}{2} b_{j k}\left|\kappa_{2-122}\right| \sin \left(2 \beta_{P R}^{j k}\right) \cos \left(\gamma_{P R}^{j k}+\xi\right),
\end{aligned}
$$

where the constant phase angle $\xi$ is defined by

$$
\xi=\arg \kappa_{2-122}+\alpha_{R L}^{0}-\omega_{r} t^{0} .
$$

The scaling factor $\kappa_{2-122}$ may be evaluated through the methods described in Ref. 49. In the case of SR26, it has a magnitude given by

$$
\left|\kappa_{2-122}\right|=0.1710 \text {. }
$$

Equation (41) shows that the magnitude of the recoupled double-quantum term depends on the Euler angle $\gamma_{P R}^{j k}$. A supercycle of this form is therefore not $\gamma$ encoded.

\section{Double-quantum filtering}

Double-quantum recoupling sequences convert longitudinal spin angular momentum into double-quantum coherence and vice versa. Signal pathways passing through double-quantum coherence may be selected by standard phase-cycling procedures. $^{53-55}$ Double-quantum filtering suppresses the signals from isolated spin-1/2 nuclei and is a common technique for improving spectral resolution and selectivity.

The efficiency of the overall process may be quantified by the double-quantum filtering efficiency (DQFE), given by

$$
f_{\mathrm{DQ}}\left(\tau_{\text {exc }}, \tau_{\text {rec }}\right)=f_{+2}\left(\tau_{\text {exc }}, \tau_{\text {rec }}\right)+f_{-2}\left(\tau_{\text {exc }}, \tau_{\text {rec }}\right),
$$

where

$$
\begin{aligned}
f_{ \pm 2}\left(\tau_{\mathrm{exc}}, \tau_{\mathrm{rec}}\right)= & \left(I_{z} \mid I_{z}\right)^{-2}\left\langle\left(I_{z} \mid U\left(\tau_{\mathrm{rec}}\right) I_{j}^{ \pm} I_{k}^{ \pm} U\left(\tau_{\mathrm{rec}}\right)^{\dagger}\right)\right. \\
& \left.\times\left(I_{j}^{ \pm} I_{k}^{ \pm} \mid U\left(\tau_{\mathrm{exc}}\right) I_{z} U\left(\tau_{\mathrm{exc}}\right)^{\dagger}\right)\right\rangle
\end{aligned}
$$

and $I_{z}=I_{j z}+I_{k z}$ is the total angular momentum operator in the direction of the field. The angular brackets denote averaging over all molecular orientations, while the round brackets indicate a projection in operator (Liouville) space, ${ }^{56}$

$$
(A \mid B)=\operatorname{Tr}\left\{A^{\dagger} B\right\} .
$$

The symbols $\tau_{\text {exc }}$ and $\tau_{\text {rec }}$ indicate the durations of the double-quantum excitation and recovery pulse sequences. An efficiency $f_{\mathrm{DQ}}=1$ would indicate perfect conversion of longitudinal magnetization into double-quantum coherence and back again into longitudinal magnetization.

The dynamics of double-quantum excitation may be treated using the cyclic commutation relationship

$$
\left[\frac{1}{2} I_{z}, I_{x}^{\mathrm{DQ}}\right]=i I_{y}^{\mathrm{DQ}}
$$

where the imaginary double-quantum operator is

$$
I_{y}^{\mathrm{DQ}}=\frac{1}{2 i}\left(I_{j}^{+} I_{k}^{+}-I_{j}^{-} I_{k}^{-}\right)
$$

Equation (47) may be used together with Eq. (38) to predict the state when the SR26 sequence is applied for a time $\tau_{\text {exc }}$ to an initial density operator proportional to $I_{z}$,

$$
\begin{aligned}
U\left(\tau_{\mathrm{exc}}\right) I_{z} U\left(\tau_{\mathrm{exc}}\right)^{\dagger} \cong & I_{z} \cos \left(\bar{\omega}_{\mathrm{SR} 26} \tau_{\mathrm{exc}}\right) \\
& -2 I_{y}^{\mathrm{DQ}} \sin \left(\bar{\omega}_{\mathrm{SR} 26} \tau_{\mathrm{exc}}\right) .
\end{aligned}
$$

The second term in this expression corresponds to excited double-quantum coherences. The theoretical double-quantum filtering efficiency for SR26 is therefore given by

$$
f_{\mathrm{DQ}}\left(\tau_{\mathrm{exc}}, \tau_{\mathrm{rec}}\right) \cong\left\langle\sin \left(\bar{\omega}_{\mathrm{SR} 26} \tau_{\mathrm{exc}}\right) \sin \left(\bar{\omega}_{\mathrm{SR} 26} \tau_{\mathrm{rec}}\right)\right\rangle
$$

\section{Symmetrical protocol}

A common procedure is to measure the double-quantumfiltered signal as a function of the durations of the excitation and reconversion sequences, which are incremented in synchrony, keeping the durations of the two sequences equal $\tau_{\text {exc }}=\tau_{\text {rec }}=\tau$. The signal trajectory obtained by this symmetrical protocol is defined

$$
f_{\mathrm{DQ}}^{\mathrm{symm}}(\tau)=f_{\mathrm{DQ}}(\tau, \tau)
$$

and is given for the case of SR26 by

$$
f_{\mathrm{DQ}}^{\mathrm{symm}}(\tau) \cong\left\langle\sin ^{2}\left(\bar{\omega}_{\mathrm{SR} 26} \tau\right)\right\rangle=\frac{1}{2}-\frac{1}{2}\left\langle\cos \left(2 \bar{\omega}_{\mathrm{SR} 26} \tau\right)\right\rangle .
$$

The second term may be written explicitly using the expressions in Eq. (41), giving

$$
\begin{aligned}
\left\langle\cos \left(2 \bar{\omega}_{\mathrm{SR} 26} \tau\right)\right\rangle= & (4 \pi)^{-1} \int_{0}^{2 \pi} d \gamma_{P R}^{j k} \int_{0}^{\pi} \sin \beta_{P R}^{j k} d \beta_{P R}^{j k} \\
& \times \cos \left(3\left|\kappa_{2-122}\right| b_{j k} \tau \sin 2 \beta_{P R}^{j k} \cos \left(\gamma_{P R}^{j k}+\xi\right)\right),
\end{aligned}
$$

where $\left\{\alpha_{P R}^{j k}, \beta_{P R}^{j k}, \gamma_{P R}^{j k}\right\}$ are Euler angles relating the reference frame of the dipole-dipole coupling between spins $I_{j}$ and $I_{k}$ to a rotor-fixed reference frame. Analytical expressions for integrals of this type have been found in the context of the REDOR experiment. ${ }^{57}$ The theoretical double-quantumfiltered trajectory for SR26, in a symmetrical incrementation protocol, is given by

$f_{\mathrm{DQ}}^{\mathrm{symm}}(\tau)=\frac{1}{2}-\frac{\sqrt{2} \pi}{8} J_{1 / 4}\left(\frac{3}{2}\left|\kappa_{2-122}\right| b_{j k} \tau\right) J_{-1 / 4}\left(\frac{3}{2}\left|\kappa_{2-122}\right| b_{j k} \tau\right)$,

where $J_{ \pm 1 / 4}$ are quarter-integer Bessel functions. ${ }^{57}$ The theoretical expression in Eq. (54)may be evaluated very rapidly and has proved to be invaluable in structure-determination procedures. $^{45,46}$

Some theoretical plots of $f_{\mathrm{DQ}}^{\mathrm{symm}}$ for a range of ${ }^{13} \mathrm{C}-{ }^{13} \mathrm{C}$ distances are shown in Fig. 2(a). In practice, these curves are damped by relaxation and dephasing mechanisms.

\section{Constant-time protocol}

An alternative protocol which is particularly useful for long-range distance estimations is to increment $\tau_{\text {exc }}$ while decrementing $\tau_{\text {rec }}$, so as to keep the total interval $T=\tau_{\text {exc }}$ $+\tau_{\text {rec }}$ constant. $^{17,25,58-60}$ In this protocol, the doublequantum-filtered signal trajectory is given for SR26 by 

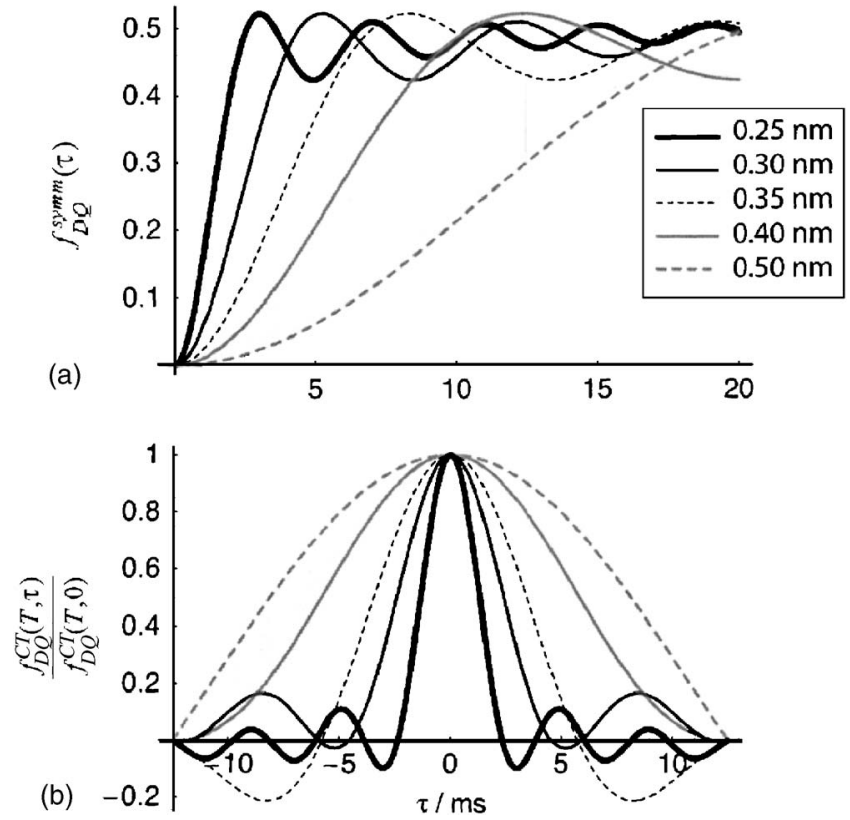

FIG. 2. Theoretical double-quantum filtering trajectories for SR26 recoupling. (a) Theoretical trajectories for the symmetric procedure, as given in Eq. (54), for a selection of ${ }^{13} \mathrm{C}-{ }^{13} \mathrm{C}$ distances. (b) Theoretical trajectories for the constant time procedure, as given in Eq. (57), for the case $T=25 \mathrm{~ms}$. Each curve is normalized against its value for $\tau=0$.

$$
f_{\mathrm{DQ}}^{\mathrm{CT}}(T, \tau)=\left\langle\sin \left(\bar{\omega}_{\mathrm{SR} 26}\left(\frac{1}{2} T+\tau\right)\right) \sin \left(\bar{\omega}_{\mathrm{SR} 26}\left(\frac{1}{2} T-\tau\right)\right)\right\rangle,
$$

where $T$ is fixed and $\tau$ takes values between $-T / 2$ and $+T / 2$. The constant-time protocol reduces the distortions to the trajectory caused by relaxation or other decay mechanisms, at the expense of a somewhat reduced signal intensity. ${ }^{17,25,58-60}$

Equation (55) may be simplified to give

$$
f_{\mathrm{DQ}}^{\mathrm{CT}}(T, \tau)=\frac{1}{2}\left\langle\cos \left(2 \bar{\omega}_{\mathrm{SR} 26} \tau\right)-\cos \left(\bar{\omega}_{\mathrm{SR} 26} T\right)\right\rangle,
$$

which has the following analytical form:

$$
\begin{aligned}
f_{\mathrm{DQ}}^{\mathrm{CT}}(T, \tau)= & \frac{\sqrt{2} \pi}{8}\left\{J_{1 / 4}\left(\frac{3}{2}\left|\kappa_{2-122}\right| b_{j k} \tau\right) J_{-1 / 4}\left(\frac{3}{2}\left|\kappa_{2-122}\right| b_{j k} \tau\right)\right. \\
& \left.-J_{1 / 4}\left(\frac{3}{4}\left|\kappa_{2-122}\right| b_{j k} T\right) J_{-1 / 4}\left(\frac{3}{4}\left|\kappa_{2-122}\right| b_{j k} T\right)\right\} .
\end{aligned}
$$

Some theoretical plots of $f_{\mathrm{DQ}}^{\mathrm{CT}}$ for a range of ${ }^{13} \mathrm{C}-{ }^{13} \mathrm{C}$ distances at $T=25 \mathrm{~ms}$ are shown in Fig. 2(b). In this case, each function has been normalized against its maximum value at $\tau=0$. The curves in Fig. 2(b) show that the zero crossings are particularly sensitive to the magnitude of the dipolar coupling.

The choice of the constant interval $T$ usually requires a compromise between optimum signal strength, which dictates a value of $T$ close to that maximizing $f_{\mathrm{DQ}}^{\mathrm{CT}}(T, 0)$, and optimal resolution of the dipolar couplings, which requires a somewhat larger value of $T$, in order to observe at least one zero crossing in the function $f_{\mathrm{DQ}}^{\mathrm{CT}}(T, \tau)$. In any case, an educated guess at the internuclear distance is necessary before the constant-time protocol may be used.

\section{NUMERICAL CALCULATIONS}

The results given above assume the convergence of the Magnus expansion and neglect higher-order terms in the $\mathrm{BCH}$ expansion on combining cycles into supercycles. We have performed numerical simulations of the spin dynamics to test the validity of these assumptions.

\section{A. Effective Hamiltonian}

The spin system propagator for any pulse sequence may be evaluated by small-step time integration of the spin evolution, i.e., numerical solution of the equation

$$
\frac{d}{d t} U\left(t, t^{0}\right)=-i \mathcal{H}(t) U\left(t, t^{0}\right)
$$

where the Hamiltonian $\mathcal{H}(t)$ contains all internal and external spin interactions, modulated by the sample rotation. In practice the numerical evaluation of $U$ was performed by using the SIMPSON simulation package. ${ }^{61}$

For a system of two coupled spin-1/2, the numerically evaluated evolution operator $U\left(\mathcal{S}_{0}\right)$ is represented by a $4 \times 4$ complex matrix $\mathbf{U}$. The matrix is diagonalized numerically leading to

$$
\mathbf{U}=\mathbf{X} \mathbf{u} \mathbf{X}^{-1} \text {, }
$$

where the columns of $\mathbf{X}$ are the eigenvectors of $\mathbf{U}$, and the diagonal elements of the diagonal matrix $\mathbf{u}$ are the corresponding eigenvalues. The matrix representation of the effective Hamiltonian is constructed by taking the logarithm of these eigenvalues,

$$
\overline{\mathbf{H}}=(-i T)^{-1} \mathbf{X}(\ln \mathbf{u}) \mathbf{X}^{-1},
$$

where $\ln \mathbf{u}$ is calculated by taking the logarithm of the diagonal elements and $T$ is the total duration of the pulse sequence. In general, the logarithm is multiple valued; however, in the present case, the effective Hamiltonian is known to be small compared to $T^{-1}$, so the smallest value of the logarithm is appropriate.

The effective Hamiltonian may be analyzed by taking projections onto any desired spin operator $Q$, using

$$
(Q \mid \overline{\mathcal{H}})=\frac{\operatorname{Tr}\left\{\mathbf{Q}^{\dagger} \overline{\mathbf{H}}\right\}}{\operatorname{Tr}\left\{\mathbf{Q}^{\dagger} \mathbf{Q}\right\}},
$$

where $\mathbf{Q}$ is the matrix representation of $Q$. Since the twospin-1/2 system supports 16 orthogonal spin operators, the effective Hamiltonian of a 2 -spin-1/2 system may therefore be represented as a point in a 16-dimensional space. For the purposes of visualization, it is usually necessary to project this point onto a set of two-dimensional subspaces.

If the calculation is repeated for many different molecular orientations, a "cloud" of points is built up in the 16dimensional operator space. The position and extent of this cloud provide insight into the nature of the effective Hamiltonian and its orientation dependence.

Two-dimensional projections of these clouds are shown for a set of different pulse sequences in Fig. 3. The left column of this figure show the projections of $\overline{\mathcal{H}}$ onto the 


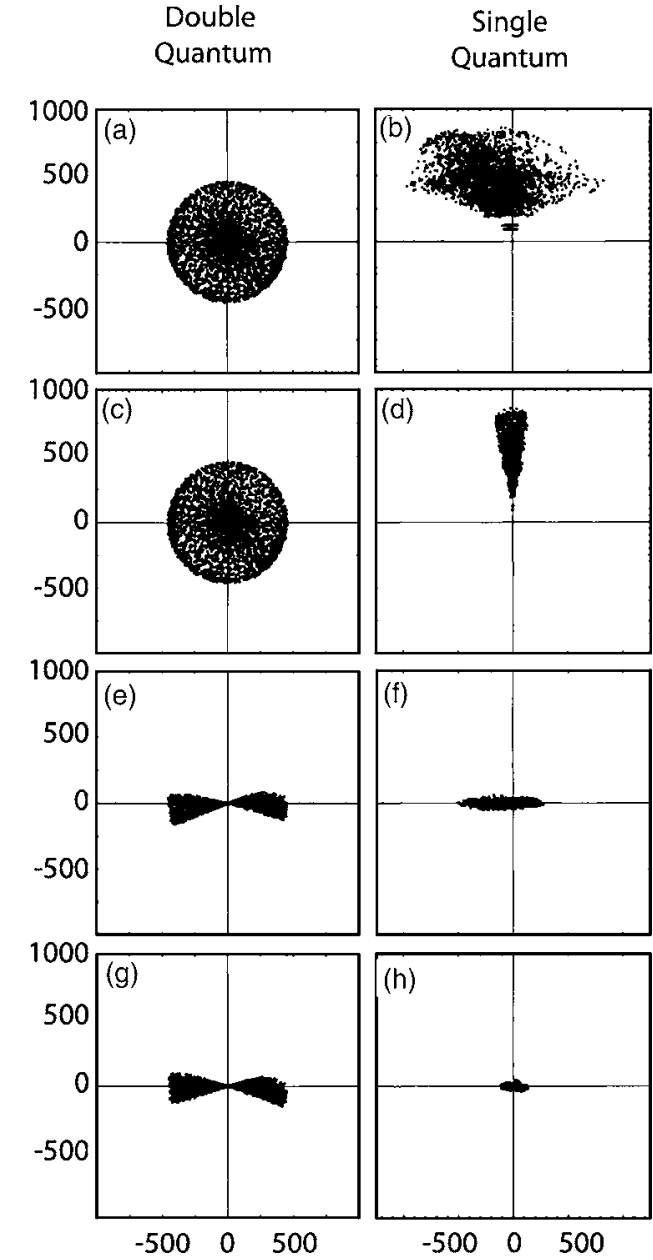

FIG. 3. Projections of the effective Hamiltonian onto two different twodimensional subspaces, for four different pulse sequences. The numerically evaluated effective Hamiltonian for each molecular orientation is represented by a point. Repetition of the calculation for many molecular orientations generates a cloud. Left column: projections onto real and imaginary double-quantum operators [Eq. (62)]. Right column: projections onto angular momentum operators along the $x$ and $z$ axes [Eq. (63)]. [(a) and (b)] A single R26 $6_{4}^{11}$ sequence. [(c) and (d)] A $\mathcal{S}_{0} \mathcal{S}_{\pi}$ supercycle. [(e) and (f)] A $\mathcal{S}_{0} \mathcal{S}_{0}^{\prime}$ supercycle. [(g) and (h) $]$ A $\mathcal{S}_{0} \mathcal{S}_{0}^{\prime} \mathcal{S}_{\pi}^{\prime} \mathcal{S}_{\pi}$ supercycle. All simulations were performed using the spin system parameters in Table I, at an external magnetic field of $9.4 \mathrm{~T}$, a spinning frequency of $7 \mathrm{kHz}$, and a rf nutation frequency of $45.5 \mathrm{kHz}$.

double-quantum operator $I_{j}^{-} I_{k}^{-}$. For each molecular orientation, a dot was placed in the two-dimensional plane at the coordinates

left column, horizontal $=\operatorname{Re}\left\{\left(I_{j}^{-} I_{k}^{-} \mid \overline{\mathcal{H}}\right)\right\}$

left column, vertical $=\operatorname{Im}\left\{\left(I_{j} I_{k} \mid \overline{\mathcal{H}}\right)\right\}$.

The right column of Fig. 3 shows the projections of $\overline{\mathcal{H}}$ onto the two single-quantum operators $I_{z}$ and $I_{x}$. For each molecular orientation, a dot was placed in the two-dimensional plane at the coordinates

right column, horizontal $=\left(I_{j x} \mid \overline{\mathcal{H}}\right)$,

right column, vertical $=\left(I_{j z} \mid \overline{\mathcal{H}}\right)$.
Figure 3 was constructed by evaluating the effective Hamiltonian over the R26 ${ }_{4}^{11}$ sequence for 3003 different values of the molecular orientation angles $\left\{\alpha_{M R}, \beta_{M R}, \gamma_{M R}\right\}$, selected by the Zaremba-Conroy-Cheng (ZCW) method. ${ }^{62,63}$ The spin system parameters used for these simulations are given in Table I. These correspond to a homonuclear 2-spin$1 / 2$ system, with large chemical shift anisotropies, and a relatively small dipole-dipole coupling, corresponding to a ${ }^{13} \mathrm{C}-{ }^{13} \mathrm{C}$ distance of around $0.3 \mathrm{~nm}$. This is a relatively challenging case. The phase shifts were assumed to be exact in all calculations.

\section{Single cycle}

Figures 3(a) and 3(b) show representations of the effective Hamiltonian for the single R26 $6_{4}^{11}$ cycle given in Eq. (4). The cloud in Fig. 3(a) corresponds to the $\gamma$-encoded doublequantum Hamiltonian $\overline{\mathcal{H}}_{\mathrm{DQ}}$. The cloud is circular in form since the phase, but not the magnitude, of $\overline{\mathcal{H}}_{\mathrm{DQ}}$ depends on the Euler angle $\gamma_{M R}$, which is a random variable in a powder. The dispersion of the cloud in the radial direction reflects the dependence of the double-quantum Hamiltonian on the angle $\beta_{M R}$.

The large diffuse cloud in Fig. 3(b) indicates the presence of high-order chemical shift terms in the effective Hamiltonian. The cloud is loosely clustered around the longitudinal axis, which reflects the dominance of the secondorder $\overline{\mathcal{H}}_{z}$ term. However, the dispersion of the cloud along the $x$ axis indicates that third-order (and higher) chemical shift terms $\overline{\mathcal{H}}_{x}$ are also significant.

For the case chosen, which has rather large chemical shift terms and a small homonuclear coupling, the "chemical shift" cloud in Fig. 3(b) is around the same size as the "double-quantum cloud" in Fig. 3(a). This suggests a severe chemical shift interference with the double-quantum recouping. This problem was represented in a different way by Karlsson et al. ${ }^{43}$

\section{The supercycle $\mathcal{S}_{0} \mathcal{S}_{\pi}$}

Figures 3(c) and 3(d) show representations of the effective Hamiltonian for the $\mathcal{S}_{0} \mathcal{S}_{\pi}$ supercycle. As anticipated from Eq. (35), the $\gamma$ encoding of the double-quantum Hamiltonian is left intact. The compression of the cloud in Fig. 3(c) shows that the supercycle reduces the $\overline{\mathcal{H}}_{x}$ terms generated by the chemical shift interference, but does not reduce the more harmful $\overline{\mathcal{H}}_{z}$ terms.

\section{The supercycle $\mathcal{S}_{0} \mathcal{S}_{0}^{\prime}$}

Figures 3(e) and 3(f) show the projected average Hamiltonian components generated by the $\mathcal{S}_{0} \mathcal{S}_{0}^{\prime}$ supercycle. The reduced vertical dispersion of the cloud in Fig. 3(f) illustrates the removal of the second-order $\overline{\mathcal{H}}_{z}$ term, in agreement with Eq. (36). In addition, the cloud in Fig. 3(f) is compressed in the horizontal direction, in comparison to that in Fig. 3(b). This corresponds to the removal of the third-order $\overline{\mathcal{H}}_{x}$ term by the $\mathcal{S}_{0} \mathcal{S}_{0}^{\prime}$ supercycle. The marked squeezing of the 
TABLE I. Spin interaction parameters used in the NMR simulations.

\begin{tabular}{lccc}
\hline \hline Interactions & Notes & Diammonium $\left[1,4-{ }^{13} \mathrm{C}_{2}\right]$-fumarate & {$\left[11,20-{ }^{13} \mathrm{C}_{2}\right]$-all-E-retinal } \\
\hline$\delta_{j}^{\text {sio }}-\delta_{k}^{\text {iso }}$ & $\mathrm{a}$ & 0 & $-121.2 \mathrm{ppm}$ \\
$\delta_{j}^{\text {aniso }}$ & $\mathrm{b}$ & $-64.8 \mathrm{ppm}$ & $15.5 \mathrm{ppm}$ \\
$\eta_{j}$ & $\mathrm{c}$ & 0.82 & 1.0 \\
$\left(\alpha_{P M}^{j}, \beta_{P M}^{j}, \gamma_{P M}^{j}\right)$ & $\mathrm{d}$ & $\left(28.9^{\circ}, 15.3^{\circ},-42.6^{\circ}\right)$ & $\left(0,5.7^{\circ}, 0\right)$ \\
$\delta_{k}^{\text {aniso }}$ & $\mathrm{b}$ & $-64.8 \mathrm{ppm}$ & $-112.2 \mathrm{ppm}$ \\
$\eta_{k}$ & $\mathrm{c}$ & 0.82 & 0.72 \\
$\left(\alpha_{P M}^{k}, \beta_{P M}^{k}, \gamma_{P M}^{k}\right)$ & $\mathrm{d}$ & $\left(28.9^{\circ}, 15.3^{\circ},-42.6^{\circ}\right)$ & $\left(-31^{\circ}, 85^{\circ},-87^{\circ}\right)$ \\
$b_{j k} / 2 \pi$ & $\mathrm{e}$ & $-136.5 \mathrm{~Hz}$ & $-290.7 \mathrm{~Hz}$ \\
$\left(\alpha_{P M}^{j k}, \beta_{P M}^{j k}, \gamma_{P M}^{j k}\right)$ & $\mathrm{f}$ & $\left(0,90^{\circ}, 0\right)$ & $(0,0,0)$ \\
\hline
\end{tabular}

${ }^{\mathrm{a}}$ Isotropic shift difference between sites.

${ }^{\mathrm{b}} \mathrm{CSA}$ (deshielding units).

${ }^{c}$ Biaxiality (asymmetry parameter).

${ }^{\mathrm{d}}$ Euler angles (degrees) relating the principal axis system of the CSA to the molecular reference frame.

${ }^{\mathrm{e}}$ Dipole-dipole coupling constant.

${ }^{\mathrm{f}}$ Euler angles (degrees) relating the principal axis system of the ${ }^{13} \mathrm{C}_{2}$ dipole-dipole coupling to the molecular reference frame.

The parameters for diammonium $\left[1,4-{ }^{13} \mathrm{C}_{2}\right]$-fumarate are adapted from Ref. 70 and employ a molecular reference frame with the $z$ axis perpendicular to the olefinic plane and the $x$ axis parallel to the line joining the two carboxylate carbon nuclei. The parameters for $\left[11,20-{ }^{13} \mathrm{C}_{2}\right]$-all- $E$-retinal are adapted from Ref. 72 and employ a molecular reference frame with the $z$ axis parallel to the line joining the two ${ }^{13} \mathrm{C}$ nuclei and the $x$ axis in the plane of the conjugated system.

double-quantum cloud in the vertical dimension, shown in Fig. 3(e), illustrates the removal of double-quantum $\gamma$ encoding.

\section{The $\mathrm{SR}_{26}^{11}$ supercycle}

Figures $3(\mathrm{~g})$ and $3(\mathrm{~h})$ show the projected average Hamiltonian components generated by the $\mathcal{S}_{0} \mathcal{S}_{0}^{\prime} \mathcal{S}_{\pi}^{\prime} \mathcal{S}_{\pi}$ supercycle. As with the $\mathcal{S}_{0} \mathcal{S}_{0}^{\prime}$ supercycle, the sequence is no longer $\gamma$ encoded. The loss of $\gamma$ encoding is an undesirable but necessary consequence of removing the chemical shift interference terms, represented by the strong radial compression of the cloud displayed in Fig. 3(b).

\section{B. Double-quantum trajectories}

Figure 4 shows numerical simulations of the doublequantum filtering efficiencies $f_{\mathrm{DQ}}^{\mathrm{symm}}(\tau)$ as a function of $\tau$, as defined in Eq. (51).

\section{Single cycles}

The simulated curves shown in Fig. 4(a) involve nonsupercycled R26 $6_{4}^{11}$ sequences. The bold solid line (i) represents an idealized case in which the dipole-dipole coupling from Table I was used, but with all chemical shift parameters set to zero. The strong oscillations with a maximum DQ efficiency of approximately $73 \%$ are characteristic of $\gamma$-encoded double-quantum recouping. ${ }^{18-23}$ The thin solid line (ii) shows a more realistic simulation in which all parameters in Table I are included. The maximum efficiency is much lower and negative double-quantum-filtered signals are generated at longer times. This is a symptom of the chemical shift interference in the average Hamiltonian, illustrated in Fig. 3(b). Similar problems with symmetry-based recouping sequences for the case of weak dipole-dipole couplings and large chemical shift terms were identified by Karlsson et al. ${ }^{43}$
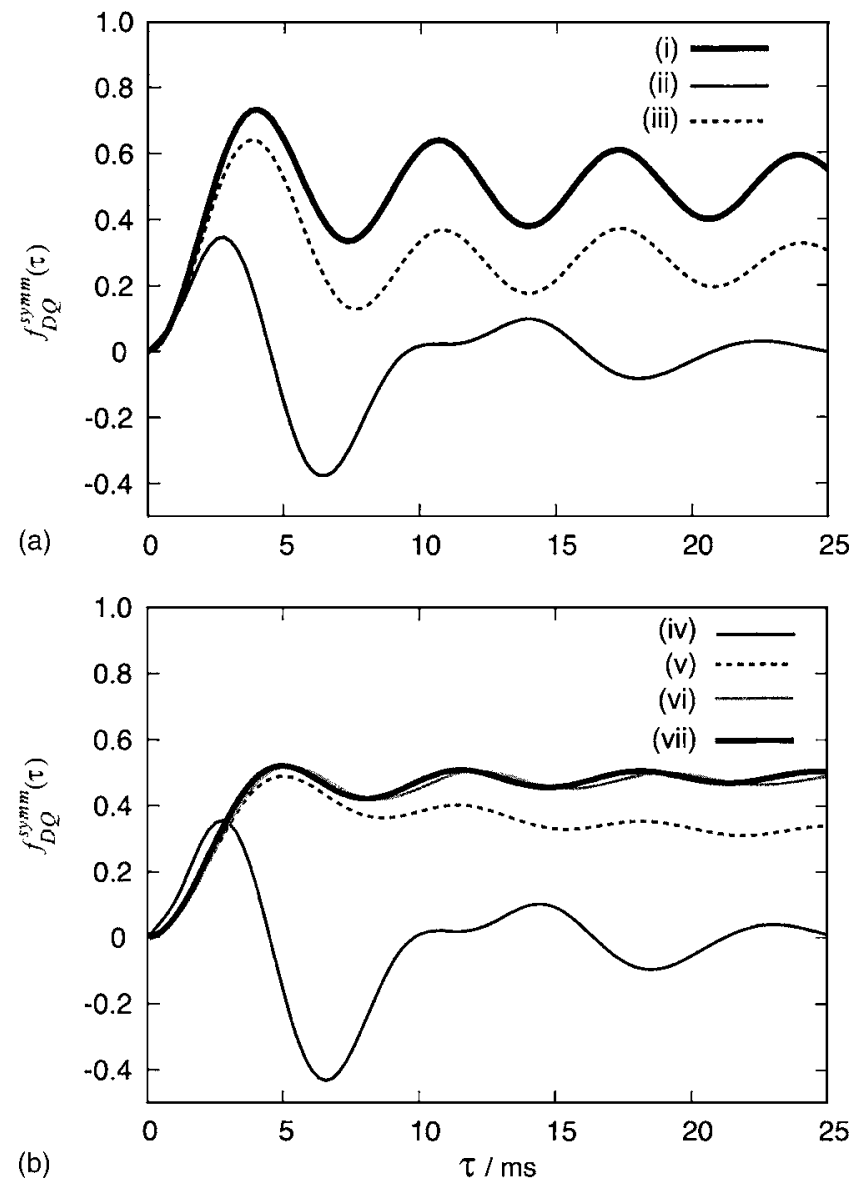

FIG. 4. Numerical simulations of symmetric double-quantum filtering trajectory functions $f_{\mathrm{DQ}}^{\mathrm{symm}}(\tau)$ as defined in Eq. (51). Unless stated, all simulations use the parameters in Table I, with a magnetic field of $9.4 \mathrm{~T}$, a spinning frequency of $7 \mathrm{kHz}$, and a rf nutation frequency of $45.5 \mathrm{kHz}$. (a) Trajectories without supercycling: (i) ideal trajectory for repetitions of the R26 $6_{4}^{11}$ sequence, omitting all chemical shift terms; (ii) calculated trajectory for R26 ${ }_{4}^{11}$ but including all parameters in Table I, (iii), as in (ii), but adjusting the rf phases by $\epsilon=-0.1^{\circ}$, as defined in Eq. (A10). (b) Trajectories for supercycles: (iv) calculated trajectory for the $\mathcal{S}_{0} \mathcal{S}_{\pi}$ supercycle; (v) calculated trajectory for the $\mathcal{S}_{0} \mathcal{S}_{0}^{\prime}$ supercycle; (vi) calculated trajectory for the SR26 ${ }_{4}^{11}$ supercycle; and (vii) result of the analytical formula in Eq. (54). 


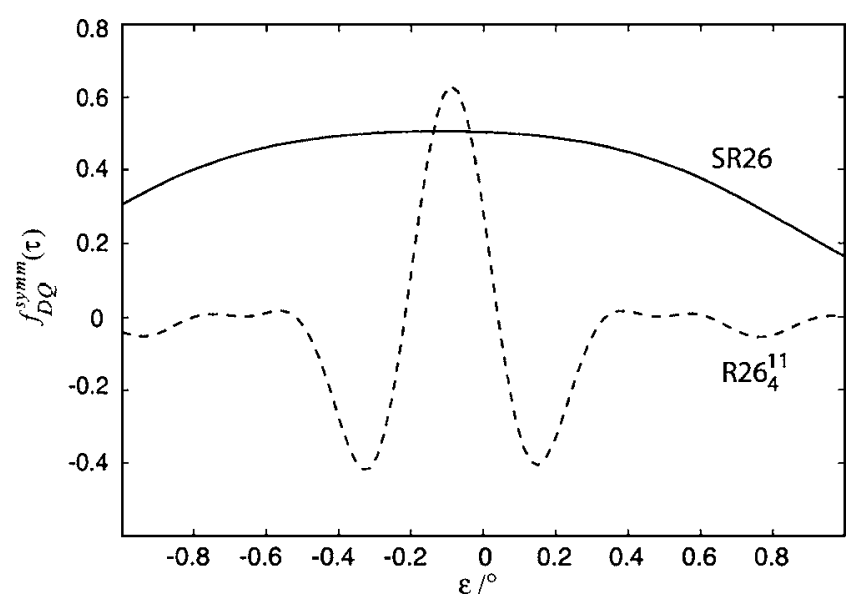

FIG. 5. Simulated double-quantum filtering efficiency as a function of the phase adjustment parameter $\epsilon$ [see Eq. (A10)]. Dashed line: simulations of $f_{\mathrm{DQ}}^{\text {symm }}(\tau)$ for the R $26_{4}^{11}$ sequence, at the point of maximum double-quantumfiltered signal $(\tau=3.43 \mathrm{~ms})$. Solid line: simulations of $f_{\mathrm{DQ}}^{\mathrm{symm}}(\tau)$ for the SR26 $6_{4}^{11}$ supercycle, at the point of maximum double-quantum-filtered signal $(\tau=4.57 \mathrm{~ms})$. All simulations were performed using the spin system parameters in Table I, at an external magnetic field of $9.4 \mathrm{~T}$, a spinning frequency of $7 \mathrm{kHz}$, and a rf nutation frequency of $45.5 \mathrm{kHz}$.

The dashed line (iii) in Fig. 4(a) shows the result of simulating $\mathrm{R}^{2} 6_{4}^{11}$ but with a small phase adjustment of $\epsilon=-0.1^{\circ}$, as defined in Eq. (73). Phase shift tuning partially compensates the chemical shift interference and leads to a double-quantum filtering trajectory which is much closer to the ideal curve. This protocol was used in order to estimate medium to long-range spin-spin distances using $\mathrm{R} 14_{2}^{6}$ recouping. ${ }^{23}$

The extreme sensitivity of the double-quantum filtering efficiency to $\mathrm{rf}$ phase shifts is explored further in Fig. 5 (dashed line). For the simulation parameters used, the double-quantum filtering efficiency of $\mathrm{R}_{2} 6_{4}^{11}$ is sensitive to phase changes of the order of $0.01^{\circ}$. Phase shifts with such accuracy are beyond the technical specifications of many commercial spectrometers. Sequences such as $\mathrm{R}_{2} 6_{4}^{11}$ and its relatives are therefore not recommended for double-quantum experiments at medium-range to long-range spin-spin distances, without the stabilization offered by supercycling.

\section{Supercycles}

The simulated curves shown in Fig. 4(b) concern various supercycles of the R26 $6_{4}^{11}$ sequence. The solid line (iv) in Fig. 4(b) shows the effect of the $\mathcal{S}_{0} \mathcal{S}_{\pi}$ supercycle. This is similar to the simulation for the nonsupercycled $\mathrm{R} 26_{4}^{11}$ sequence [line (ii) in Fig. 4(a)], confirming that the $\mathcal{S}_{0} \mathcal{S}_{\pi}$ supercycle does not provide any significant improvement in performance.

The construction of a $\mathcal{S}_{0} \mathcal{S}_{0}^{\prime}$ supercycle, on the other hand, immediately leads to a considerable improvement of the long-term performance of the double-quantum pulse sequence [line (v) in Fig. 4(b)]. However, this long-term improvement is accompanied by the classic symptoms of loss in $\gamma$ encoding. The dipolar oscillations are reduced in amplitude and the maximum achievable efficiency is around 50\% rather than the $\gamma$-encoded optimum of $73 \%$.<smiles>CC1=C(/C=C/C(C)=C/OC=C(O)C=O)C(C)(C)CCC1</smiles>

(a)

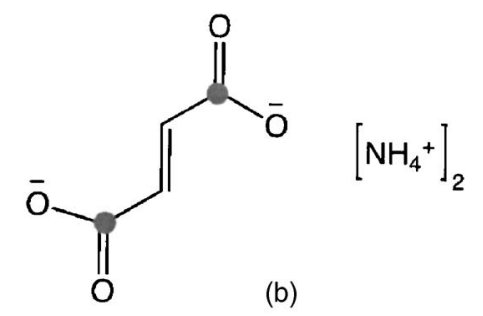<smiles>[NH3+]CC(=O)[O-]</smiles>

(c)

FIG. 6. Molecular systems used in the experimental demonstrations. Gray circles indicate ${ }^{13} \mathrm{C}$ labels. (a) $\left[11,20-{ }^{13} \mathrm{C}_{2}\right]$-all- $E$-retinal, (b) diammonium $\left[1,4-{ }^{13} \mathrm{C}_{2}\right]$-fumarate, and (c) $\left[1-{ }^{13} \mathrm{C}\right]$-glycine.

Construction of the $\mathcal{S}_{0} \mathcal{S}_{0}^{\prime} \mathcal{S}_{\pi}^{\prime} \mathcal{S}_{\pi}$ supercycle (SR26) leads to further stabilization of the sequence at long excitation times [line (vi) in Fig. 4(b)]. The double-quantum-filtered signal trajectory for the SR26 supercycle is very close to the analytical form given in Eq. (54), which is shown by the solid bold line (vii).

The SR26 supercycle is also much more stable than the single $\mathrm{R}_{2} 6_{4}^{11}$ cycle with respect to phase-shift errors, as illustrated in Fig. 5 (solid line). The required phase accuracy for the supercycle is around $\sim 0.5^{\circ}$, which is readily achievable on most commercial instruments. Note, however, that the achievable double-quantum signal is less than that predicted for the single cycle after optimization of the phase shift. This drop in optimum efficiency is associated with the loss of $\gamma$ encoding for the supercycle.

\section{METHODS}

\section{A. Samples}

The experimental demonstrations employed three different ${ }^{13} \mathrm{C}$-labeled organic compounds, with molecular structures shown in Fig. 6.

$\left[11,20-{ }^{13} \mathrm{C}_{2}\right]$-all-E-retinal [Fig. 6(a)] was used to demonstrate the determination of internuclear distances. The ${ }^{13} \mathrm{C}$-labeled retinal was synthesized by standard methods, ${ }^{64}$ and recrystallized from $n$-pentane, together with a tenfold excess of nonlabeled all- $E$-retinal. The distance between the two ${ }^{13} \mathrm{C}$ nuclei, determined by $\mathrm{x}$-ray diffraction, ${ }^{65}$ is $295.9 \mathrm{pm}$.

The estimation of relative shielding tensor orientations was demonstrated using diammonium $\left[1,4-{ }^{13} \mathrm{C}_{2}\right]$-fumarate (DAF) [Fig. 6(b)]. The ${ }^{13} \mathrm{C}_{2}$-labeled diammonium fumarate was diluted to a level of $6 \%$ in nonlabeled diammonium fumarate. The distance between the two ${ }^{13} \mathrm{C}$ nuclei, determined by $\mathrm{x}$-ray diffraction, ${ }^{66}$ is $387.8 \mathrm{pm}$. 


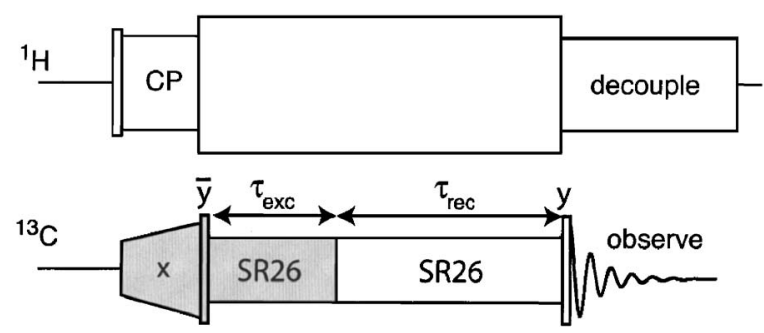

(a)

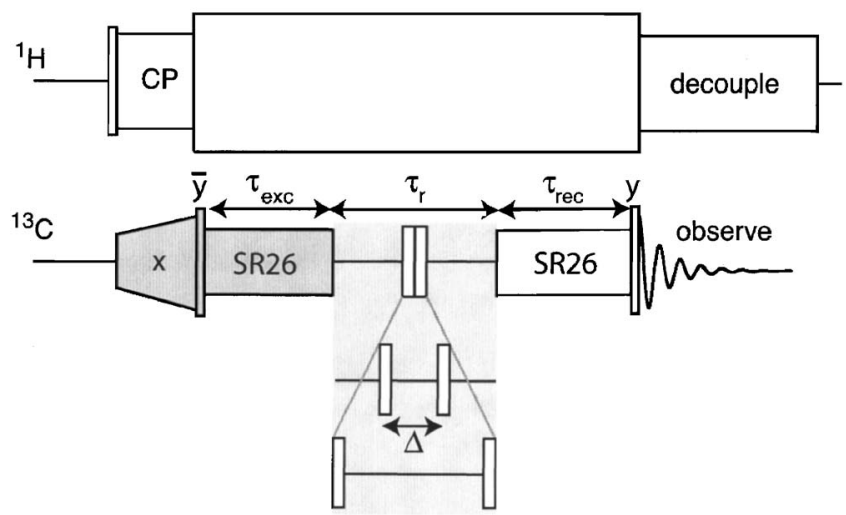

(b)

FIG. 7. Pulse sequences for the application of SR 26 to the ${ }^{13} \mathrm{C}$ spectroscopy of organic solids. (a) Pulse sequence for the double-quantum filtering of cross-polarized ${ }^{13} \mathrm{C}$ NMR signals. The shaded elements are given a four-step phase cycle to select signals passing through $( \pm 2)$-quantum coherence. The thin rectangles represent $\pi / 2$ pulses. (b) Pulse sequence for the determination of relative CSA tensor orientations. A single rotor period of doublequantum evolution is inserted, interrupted by two strong $\pi$ pulses, and separated by an interval $\Delta$ (white rectangles). A series of experiments is performed in which $\Delta$ is increased, moving the $\pi$ pulses from the center of the rotational period $(\Delta=0)$ to the ends of the rotational period $\left(\Delta=\tau_{r}\right)$.

The demonstrations of high-order multiple-quantum excitation in solids were performed using $\left[1-{ }^{13} \mathrm{C}\right]$-glycine, used as purchased from Cambridge Isotope Laboratories (Andover, MA). The x-ray crystal structure of glycine ${ }^{67}$ shows that the $1-{ }^{13} \mathrm{C}$ sites are arranged in pairs, with an internuclear distance of $310.4 \mathrm{pm}$ within each pair. The closest approach of $1-{ }^{13} \mathrm{C}$ sites belonging to different pairs is $405.5 \mathrm{pm}$.

\section{B. NMR experiments}

All experiments were performed at a magnetic field of 14.1 T using a Varian Infinity+console, using $3.2 \mathrm{~mm}$ zirconia rotors in a standard T3 triple-resonance probehead. Data processing was performed using Varian SPINSIGHT software and MATNMR. ${ }^{68}$

All NMR experiments were performed using a spinning frequency of $5.923 \mathrm{kHz}$. The SR26 pulse sequences were performed using simultaneous ${ }^{13} \mathrm{C}$ and ${ }^{1} \mathrm{H}$ rf fields, with amplitudes corresponding to nutation frequencies of 38.5 and $100 \mathrm{kHz}$, respectively. ${ }^{13} \mathrm{C}$ signal acquisition was performed in the presence of two-pulse-phase-modulated (TPPM) ${ }^{1} \mathrm{H}$ decoupling, ${ }^{69}$ with a nutation frequency of $84 \mathrm{kHz}$. The ${ }^{13} \mathrm{C}$ reference frequency was always set to the mean resonance frequency of the recoupled sites. ${ }^{13} \mathrm{C} \pi$ pulses used a nutation frequency of $62.5 \mathrm{kHz}$.

All multiple-quantum filtering efficiencies were deter-

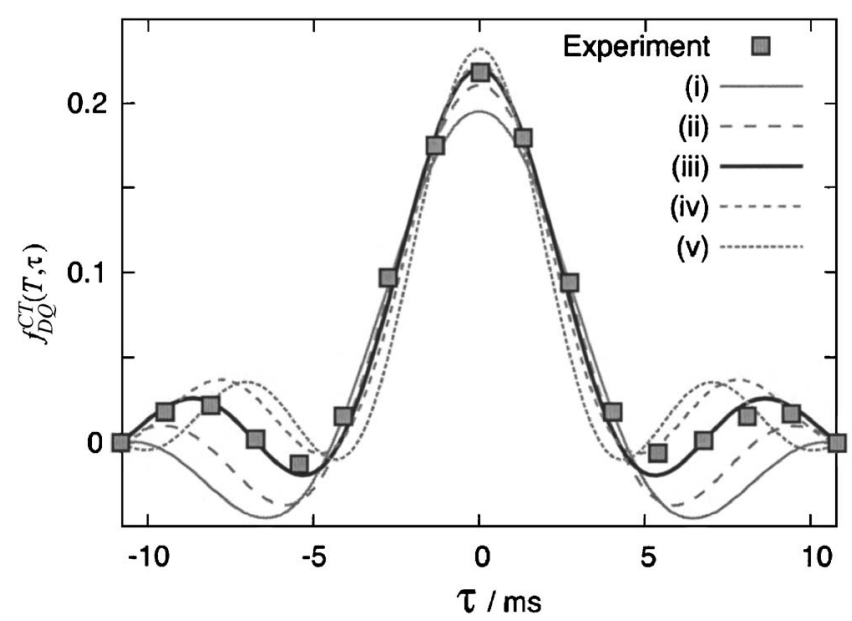

FIG. 8. Double-quantum-filtered signal amplitudes for $\left[11,20-{ }^{13} \mathrm{C}_{2}\right]$-all$E$-retinal using the SR26 supercycle. The excitation interval $\tau_{\text {exc }}$ was incremented in steps of a half-supercycle, while the reconversion interval $\tau_{\text {rec }}$ was decremented at the same time to keep the total interval fixed at $T$ $=21.6 \mathrm{~ms}$. The experimental amplitudes (gray curves) are compared with the analytical functions in Eq. (57), adjusting the vertical scale of the analytical functions in each case to obtain the best fit. The dipole-dipole couplings and corresponding internuclear distances $\left(b_{j k} / 2 \pi, r_{j k}\right)$ are as follows: (i) $(-339.1 \mathrm{~Hz}, 281.9 \mathrm{pm})$; (ii) $(-305.5 \mathrm{~Hz}, 291.9 \mathrm{pm})$; (iii) $(-276.1 \mathrm{~Hz}$, $301.9 \mathrm{pm})$; (iv) $(-250.3 \mathrm{~Hz}, 311.9 \mathrm{pm})$; and (v) $(-248.0 \mathrm{~Hz}, 312.9 \mathrm{pm})$. The bold line (iii) (corresponding to $301.9 \mathrm{pm}$ ) is the best fit.

mined by comparing the integrated multiple-quantum-filtered signals with the integrals of cross-polarized signals, obtained under identical conditions.

\section{APPLICATIONS}

\section{A. Determination of ${ }^{13} \mathrm{C}-{ }^{13} \mathrm{C}$ distances}

Internuclear distances within isolated spin pairs may be estimated by using the pulse sequence in Fig. 7(a). The DQfiltered signal amplitudes are estimated as a function of the intervals $\tau_{\text {exc }}$ and $\tau_{\text {rec }}$, which may be varied according to one of the protocols discussed above. The dipole-dipole coupling is estimated by comparing the DQ-filtered signal trajectories with the analytical functions given in Eqs. (54) and (57), or with numerical simulations. It is usually preferable to use the analytical functions, since they may be calculated very rapidly. However, one should first verify the close resemblance of the analytical curves with accurate numerical simulations for some representative cases.

Figure 8 shows experimental double-quantum-filtered signal amplitudes for $\left[11,20-{ }^{13} \mathrm{C}_{2}\right]$-all- $E$-retinal, obtained using the constant-time protocol with $T=21.6 \mathrm{~ms}$. The best fit of the analytical function in Eq. (57) to the experimental points yields a dipole-dipole coupling of $b_{j k} / 2 \pi$ $=-276 \pm 5 \mathrm{~Hz}$, which corresponds to an internuclear distance of $302 \pm 2 \mathrm{pm}$. If the fitting is repeated using SIMPSON numerical simulations rather than analytical expressions, the result is only slightly different $(297 \pm 2.4 \mathrm{pm})$.

The internuclear distance as determined by $\mathrm{x}$-ray diffraction ${ }^{65}$ is $295.9 \mathrm{pm}$. A small systematic discrepancy between NMR and x-ray diffraction of around $3 \mathrm{pm}$ is typical and may be attributed to the influence of molecular vibrations. ${ }^{23}$ 


\section{B. Determination of relative CSA orientations}

The evolution of DQ coherences is sensitive to the relative orientation of spin interaction tensors. Since the spin interaction tensors often have a known relationship with the local molecular geometry, this phenomenon often allows molecular geometrical parameters, such as torsional angles, to be determined by studying the double-quantum evolution. ${ }^{12,27-33}$ In particular, the torsional angles of a protein backbone may be estimated by determining the relative orientation of ${ }^{13} \mathrm{C}$ CSA tensors for neighboring carboxyl sites. $^{32,33}$

The SR26 pulse sequence should be very useful for such studies since it is capable of exciting double-quantum coherences between ${ }^{13} \mathrm{C}$ nuclei experiencing large chemical shift anisotropies, even at rather large internuclear distances. The evolution of the DQ coherence under the two ${ }^{13} \mathrm{C}$ CSA interactions may be studied by using the pulse sequence in Fig. 7(b), which assumes that the reference frequency is set to the mean resonance frequency of the two sites. The ${ }^{13} \mathrm{C}_{2}$ DQ coherences are excited in the usual way by a SR26 pulse sequence. The double-quantum coherences are allowed to evolve for one rotor period $\tau_{r}$, in the presence of proton decoupling, before reconversion into observable signal by a second SR26 interval. Two strong $\pi$ pulses are inserted in the evolution period, centered at positions $\tau_{r} / 2 \pm \Delta / 2$. A series of experiments is conducted in which the $\pi$-pulse separation is incremented from $\Delta=0$ (in which case the two $\pi$ pulses coincide at the center of the rotor period) to $\Delta=\tau_{r}$ (in which case the $\pi$ pulses are at the beginning and end of the rotor period). The opposed motion of the two $\pi$ pulses is depicted in Fig. 7(b).

When $\Delta=0$, the two $\pi$ pulses coincide at the center of the rotor period and hence have a little effect, since the combined flip angle is then $2 \pi$. In this case, the double-quantum coherences do not evolve at all under the $\tau_{r}$ period (except for relaxation), since the effect of the chemical shift anisotropy vanishes over the complete magic-angle rotation, and the isotropic chemical shift also has no effect, due to the choice of the spectrometer reference frequency. The chemical shift evolution also vanishes for $\Delta=\tau_{r}$, since the two $\pi$ pulses are then located at the beginning and end of the inserted rotor period. However, at intermediate values of $\Delta$, the double-quantum coherences are strongly phase modulated by the combined effects of the two CSA interactions. The double-quantum-filtered signal should therefore be maximal for $\Delta=0$ and $\Delta=\tau_{r}$, dipping strongly at intermediate values.

The precise form of the $\Delta$ dependence depends on the orientation of the two CSA tensors with respect to each other, since the DQ evolution frequency is given by the instantaneous sum of the two chemical shifts. Less intuitively, the $\Delta$ dependence of the DQ-filtered signal also depends on the orientation of the CSA tensors with respect to the molecular reference frame, even when the the principal axis systems of the two CSA tensors are held in the same relative orientation. This effect is not due to double-quantum evolution, but instead to the orientation dependence of the double-quantum excitation.

We have demonstrated this phenomenon by using SR26
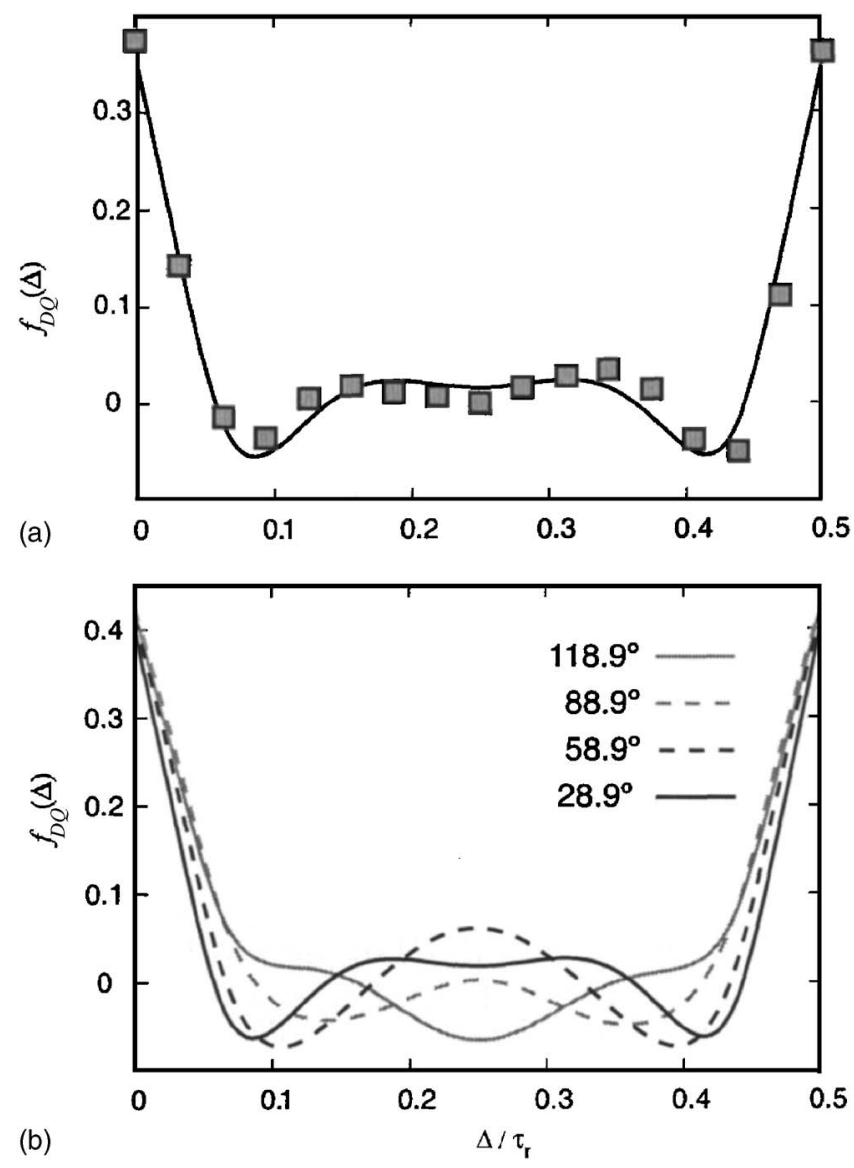

FIG. 9. Double-quantum evolution trajectories as a function of the separation $\Delta$ between the $\pi$ pulses in Fig. 7(b). All simulations and experiments use double-quantum excitation and reconversion intervals of $\tau_{\mathrm{exc}}=\tau_{\text {rec }}$ $=8.1 \mathrm{~ms}$. (a) Experimental data points for diammonium $\left[1,4-{ }^{13} \mathrm{C}_{2}\right]$-fumarate (gray squares) and simulated double-quantum trajectory for the parameters given in the first column in Table I. (b) Simulated double-quantum evolution trajectories for the parameters given in the first column in Table I, except for the Euler angles $\alpha_{P M}^{j}=\alpha_{P M}^{k}$, which are specified in the plot.

to excite double-quantum coherence between the two ${ }^{13} \mathrm{C}$ sites of diammonium $\left[1,4-{ }^{13} \mathrm{C}_{2}\right]$-fumarate, which are separated by $382 \mathrm{pm}$ [Fig. 6(b)]. In this particular case, the principal axis systems of the two CSA tensors are constrained to be collinear, due to the inversion symmetry of the crystal structure. The orientation of the two CSA tensors in a molecular reference frame has been determined by Bechmann $e t$ $a l$. by combining data from several different experiments. ${ }^{70}$ The spin system parameters are listed in the first column of Table I. For the purposes of the current study, the tensor orientations are specified using a molecular reference frame with the $z$ axis perpendicular to the plane of the olefinic system, and the $x$ axis along the line joining the two ${ }^{13} \mathrm{C}$ labels. The principal axis systems of the two CSA tensors coincide, with the most-shielded axis approximately perpendicular to the carboxylate planes.

Figure 9(a) compares the experimental $\Delta$ dependence of the DQ-filtered signal with simulations for the literature tensor orientations. ${ }^{70}$ These data were obtained using doublequantum excitation and reconversion intervals of $\tau_{\text {exc }}=\tau_{\text {rec }}$ $=8.1 \mathrm{~ms}$. The agreement is good.

Figure 9(b) shows the dependence of the $\Delta$-dependent trajectory on the CSA orientations. In these simulations, two 
of the Euler angles defining the orientation of each CSA tensor are held equal to the literature values $\left(\beta_{P M}^{j}=\beta_{P M}^{k}\right.$ $=15.3^{\circ}$ and $\left.\gamma_{P M}^{j}=\gamma_{P M}^{k}=-42.6^{\circ}\right)$, while the other Euler angles is varied with respect to the literature value $\left(\alpha_{P M}^{j}=\alpha_{P M}^{k}\right.$ $\left.=28.9^{\circ}\right)$ in steps of $30^{\circ}$. Since the Euler angle sets $\left\{\alpha_{P M}^{j}, \beta_{P M}^{j}, \gamma_{P M}^{j}\right\}$ and $\left\{\alpha_{P M}^{k}, \beta_{P M}^{k}, \gamma_{P M}^{k}\right\}$ are always equal, each simulation employs the same relative orientation of the two CSA tensors. Nevertheless, the DQ trajectories depend rather strongly on the value of $\alpha_{P M}^{j}=\alpha_{P M}^{k}$. As mentioned before, this phenomenon is due to the orientation dependence of the DQ excitation. More detailed analysis (not shown) indicates that the double-quantum experiment is able to determine the angle $\alpha_{P M}^{j}=\alpha_{P M}^{k}$ with an accuracy of approximately $\pm 10^{\circ}$.

\section{Multiple-quantum spin counting}

If a sample containing a network of coupled spins is allowed to evolve under the homonuclear dipolar couplings, Zeeman order is transformed into spin-spin correlation terms of increasingly high order, including multiple-quantum coherences. Since isolated clusters of $N$ coupled spin-1/2 only support multiple-quantum coherences of order $p$ satisfying $-N \leqslant p \leqslant N$, the detection of a NMR signal that has passed through multiple-quantum coherence of order $p$ sets a lower bound on the size of the coupled spin cluster, $N \geqslant|p|$. This technique is known as multiple-quantum spin counting. ${ }^{32,36-42}$

Initial applications of multiple-quantum spin counting concerned coupled ${ }^{1} \mathrm{H}$ nuclei, since the relatively small chemical shift anisotropies and strong homonuclear couplings make the excitation of high-order multiple-quantum coherences easier. ${ }^{37,38,40}$ The introduction of shift-compensed pulse sequences allowed applications to nuclei such as ${ }^{13} \mathrm{C}$ as well. ${ }^{32,39}$ These experiments were performed on nonrotating samples, such as ${ }^{13} \mathrm{C}$-labeled Alzheimer's peptides, where the dynamics of the multiple-quantum excitation could be analyzed to obtain information on the molecular organization. $^{32}$

It is clearly desirable to combine multiple-quantum spin counting experiments with the high resolution and sensitivity offered by magic-angle spinning. Oyler and Tycko used finite-pulse radio-frequency-driven recoupling ${ }^{16}$ (fpRFDR) to obtain multiple-quantum excitation of orders up to 8 (and possibly 10) in ${ }^{13} \mathrm{C}$-labeled amino acids. ${ }^{41}$ In this section we report unambiguous excitation of ${ }^{13} \mathrm{C}$ multiple-quantum orders up to 10 in $\left[1-{ }^{13} \mathrm{C}\right]$-glycine, using SR26.

The multiple-quantum spin counting experiments are conducted using the pulse sequence in Fig. 7(a). The durations of the excitation and reconversion blocks are equal $(\tau$ $=\tau_{\text {exc }}=\tau_{\text {rec }}$ ). A series of $\mathcal{N}$ separate experiments is conducted in which the overall phase shift $\Phi$ of the excitation block (shaded) is incremented from 0 to $2 \pi(\mathcal{N}-1) / \mathcal{N}$ in steps of $2 \pi / \mathcal{N}$. In order to prevent folding, the number of phase steps $\mathcal{N}$ should exceed twice the maximal excited coherence order. The data are stored separately for each value of $\Phi$. Fourier transformation with respect to $\Phi$ generates a multiplequantum excitation spectrum which displays the fraction of signal $f_{ \pm p}(\tau)$ passing through multiple-quantum orders $\pm p$ at the junction of the excitation and reconversion blocks. Multiple-quantum excitation spectra for a set of $\tau$ values are shown for the case of SR26 applied to $\left[1-{ }^{13} \mathrm{C}\right]$-glycine in the left-hand column of Fig. 10.

In practice, experiments with different $\Phi$ values were executed in random order to avoid systematic errors associated with incomplete relaxation between transients.

The left-hand column of Fig. 10 shows the multiplequantum excitation spectra for SR26 applied to $\left[1-{ }^{13} \mathrm{C}\right]$-glycine, at a set of recoupling times $\tau$ in the range of 5.4-27 ms. In all cases, only even-quantum coherences have a significant amplitude, as expected for double-quantum recoupling. The presence of multiple-quantum orders up to 10 is clearly visible at long times [Figs. 10(c) and 10(d)]. The multiple-quantum-filtered intensities $f_{ \pm p}(\tau)$ are normalized against the total signal intensity obtained in an ordinary cross-polarization experiment. Figure 10(a) shows that almost $50 \%$ of the $\left[1-{ }^{13} \mathrm{C}\right]$-glycine signal passes through intermolecular double-quantum coherence when SR26 is applied for $5.4 \mathrm{~ms}$. Figure 10(c) shows that almost $5 \%$ of the [1 $\left.-{ }^{13} \mathrm{C}\right]$-glycine signal passes through intermolecular fourquantum coherence when SR26 is applied for $21.6 \mathrm{~ms}$.

The right-hand column of Fig. 10 shows the same data on a logarithmic scale, in order to accentuate the small peaks. Gray shading indicates negative values, which were inverted in sign before taking the logarithm. The error bars were obtained from the distribution of intensities obtained for six repetitions of the same experiment. These plots clearly show the "noise floor" of the multiple-quantum excitation data and establish that the ten-quantum peaks in Figs. 10(c) and 10(d) are significant.

Recently, an extension of the multiple-quantum spin counting experiment known as spherical tensor analysis (STA) has been demonstrated. ${ }^{71}$ In this experiment, the spin order terms are classified according to their rotational properties about three orthogonal axes. STA experiments on $\left[1-{ }^{13} \mathrm{C}\right]$-glycine using SR26 demonstrated the presence of spherical tensor spin operators up to rank 4. The fourth-rank spin operators appear more rapidly than four-quantum coherences. $^{71}$

\section{CONCLUSIONS}

Symmetry-based pulse sequences provide a set of convenient and versatile tools for a variety of recoupling tasks. ${ }^{19-26}$ They are particularly well established for recoupling of strong dipolar interactions, since in this context they have two distinct advantages over most other pulse sequences: (i) they allow a straightforward implementation of $\gamma$-encoded recoupling, which provides strong dipolar oscillations with a high dynamic range, and (ii) they permit a good time resolution of the dipolar oscillations, since the recoupling intervals may be incremented in intervals of much less than one rotor period. ${ }^{26}$

Symmetry-based recoupling sequences have been less suitable for recoupling over longer distances, since they are sensitive to interference from chemical shift anisotropy and phase-shift errors. $^{23,43}$ The work described in this paper shows that these disadvantages may be avoided by the con- 
$f_{ \pm p}(\tau)$

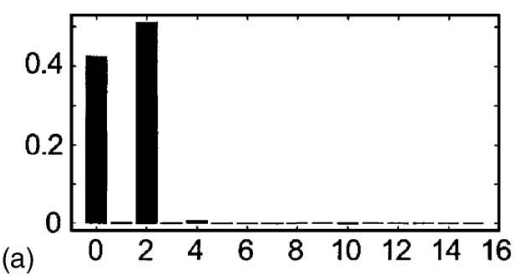

(a)

(b)
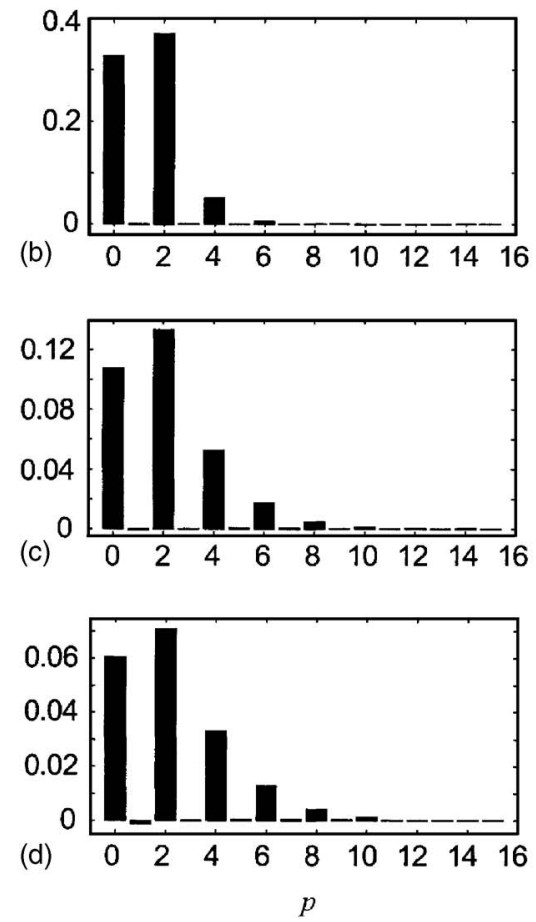

$\log _{10}\left|f_{ \pm p}(\tau)\right|$
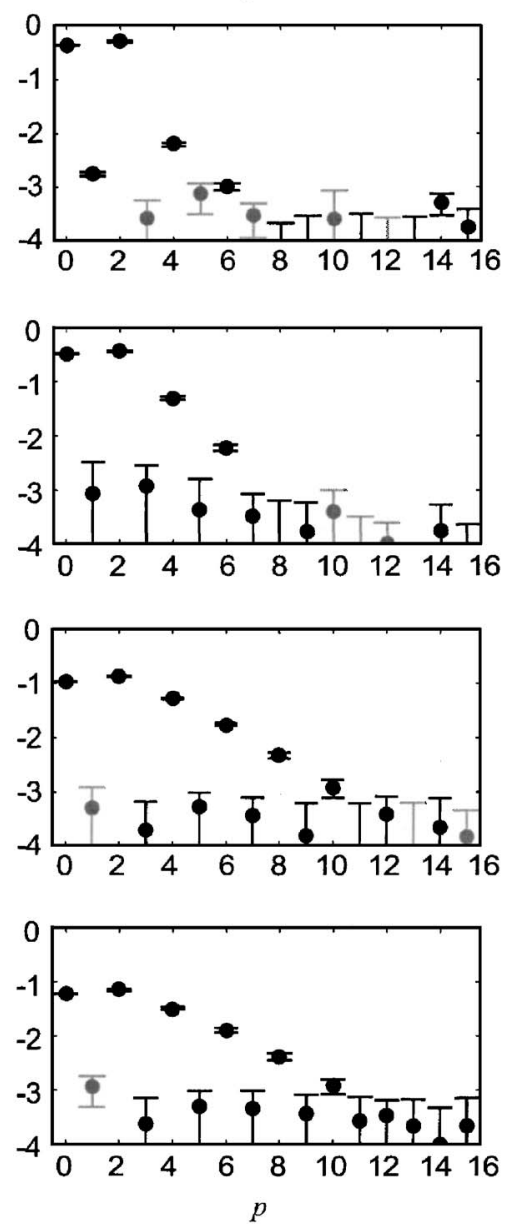

FIG. 10. Multiple-quantum-filtered ${ }^{13} \mathrm{C}$ signal amplitudes for $\left[1-{ }^{13} \mathrm{C}\right]$-glycine using SR26 recoupling sequences of different durations. Left column: multiplequantum-filtered signal amplitudes, relative to a crosspolarization experiment. Right column: representation of the same data on a logarithmic plot, using gray coloring to represent negative values, which were inverted before taking the logarithm. The error bars in the righthand column were estimated by analyzing the data from a set of identical experiments (see text). The recoupling durations are the same for the excitation and reconversion sequences $\left(\tau_{\text {exc }}=\tau_{\text {rec }}\right)$ and are as follows: (a) 5.4, (b) 10.8 (c) 21.6 , and (d) $27.0 \mathrm{~ms}$. struction of supercycles. Supercycles of the form $\mathcal{S}_{0} \mathcal{S}_{0}^{\prime} \mathcal{S}_{\pi}^{\prime} \mathcal{S}_{\pi}$ are particularly useful. The supercycle SR26, which is based on the $\mathrm{R}_{2} 6_{4}^{11}$ symmetry-based cycle, provides robust doublequantum recoupling which is well compensated for chemical shift anisotropy and phase-shift errors. The results shown in this paper demonstrate accurate ${ }^{13} \mathrm{C}-{ }^{13} \mathrm{C}$ distance measurements and high-order intermolecular multiple-quantum excitation. The double-quantum coherences excited by SR26 may be used to determine the relative orientations of chemical shift anisotropy tensors.

In the case of isolated spin-1/2 pairs, the trajectories of the double-quantum-filtered signals are often given to a good approximation by Bessel function analytical expressions [Eqs. (54) and (57)]. These functions may be computed very rapidly, allowing the rapid optimization of molecular structural parameters against the experimental double-quantumfiltered signals. Several zeolite framework structures have been solved by analyzing the ${ }^{29} \mathrm{Si}-{ }^{29} \mathrm{Si}$ double-quantum trajectories induced by SR26 recoupling. ${ }^{45,46}$

Supercycles such as SR26 do have some disadvantages over single symmetry-based sequences such as $\mathrm{C} 7{ }_{2}^{1}, \mathrm{R} 26_{4}^{11}$, and $\mathrm{R} 14_{2}^{6}$. The loss of $\gamma$ encoding leads to a lower achievable double-quantum efficiency than for simple cycles, and much lower dynamic range in the dipolar oscillations. In addition, the time resolution of the recoupling intervals is greatly reduced since an integer multiple of supercycles must be com- pleted (although sometimes the completion of a fractional supercycle is acceptable). For example, SR26 consists of a $\mathcal{S}_{0} \mathcal{S}_{0}^{\prime} \mathcal{S}_{\pi}^{\prime} \mathcal{S}_{\pi}$ supercycle. Completion of a half-supercycle is acceptable since the $\mathcal{S}_{0} \mathcal{S}_{0}^{\prime}$ supercycle has the same orientationdependent recoupling characteristics as the full supercycle. However, completion of a quarter supercycle is not acceptable since $\mathcal{S}_{0}$ is $\gamma$ encoded while $\mathcal{S}_{0} \mathcal{S}_{0}^{\prime} \mathcal{S}_{\pi}^{\prime} \mathcal{S}_{\pi}$ is not. The minimum incrementation interval for SR26 is therefore eight rotor periods. This may be contrasted with the basic cycle $\mathrm{R} 26_{4}^{11}$, for which the minimum incrementation interval is two $R$ elements, i.e., $4 / 13$ of a rotor period.

For these reasons, supercycles such as SR26 are only to be preferred over single symmetry-based cycles in the case of weak dipolar interactions. For such cases, the reduced time resolution of supercycles is not a serious issue and their increased robustness at long times is a decisive advantage.

\section{ACKNOWLEDGMENTS}

This research was supported by the ERSRC (UK) and the NSF (Norway). The authors would like to thank H. Luthman for synthesis of the $\left[11,20-{ }^{13} \mathrm{C}_{2}\right]$-all- $E$-retinal, A. Sebald for loaning the DAF sample, and O. G. Johannessen for instrumental support. 


\section{APPENDIX A: rf PROPAGATORS OF RN ${ }_{n}^{\nu}$ SEQUENCES}

The spin Hamiltonian contains terms for the internal spin interactions, and the applied radio-frequency pulse sequence,

$$
\mathcal{H}(t)=\mathcal{H}_{\text {int }}(t)+\mathcal{H}_{\mathrm{rf}}(t)
$$

A propagator is defined by integrating the effect of the $\mathrm{rf}$ field from the start of the pulse sequence,

$$
\frac{d}{d t} U_{\mathrm{rf}}\left(t, t^{0}\right)=-i \mathcal{H}_{\mathrm{rf}}(t) U_{\mathrm{rf}}\left(t, t^{0}\right) .
$$

The internal spin interactions are transformed into the interaction frame of the rf field,

$$
\tilde{\mathcal{H}}_{\text {int }}(t)=U_{\text {rf }}\left(t^{0}, t\right) \mathcal{H}_{\text {int }}(t) U_{\text {rf }}\left(t, t^{0}\right),
$$

where $U_{\mathrm{rf}}\left(t^{0}, t\right)=U_{\mathrm{rf}}\left(t, t^{0}\right)^{\dagger}$. The propagator over the pulse sequence may then be expressed as

$$
U\left(t, t^{0}\right)=U_{\mathrm{rf}}\left(t, t^{0}\right) \exp \left\{-i\left(t-t^{0}\right) \overline{\mathcal{H}}\left(t^{0}, t\right)\right\},
$$

where the effective Hamiltonian $\overline{\mathcal{H}}$ is expressed as a Magnus expansion

$$
\overline{\mathcal{H}}\left(t, t^{0}\right)=\overline{\mathcal{H}}^{(1)}\left(t, t^{0}\right)+\overline{\mathcal{H}}^{(2)}\left(t, t^{0}\right)+\cdots,
$$

where the first term is the average Hamiltonian,

$$
\overline{\mathcal{H}}^{(1)}\left(t, t^{0}\right)=\left(t-t^{0}\right)^{-1} \int_{t^{0}}^{t} d t^{\prime} \tilde{\mathcal{H}}_{\text {int }}\left(t^{\prime}\right) .
$$

Equation (A4) shows that the spin system propagator involves the integrated effect of the rf field over the pulse sequence, as expressed by the propagator $U_{\mathrm{rf}}$, as well as the average Hamiltonian terms $\overline{\mathcal{H}}^{(1)}, \overline{\mathcal{H}}^{(2)}$, etc.

By definition, the basic element $\mathcal{R}^{0}$ rotates the resonant spins by an odd multiple of $\pi$ around the $x$ axis,

$$
U_{\mathrm{rf}}\left(\mathcal{R}^{0}\right)= \pm \Pi_{x},
$$

where the rotation operator is

$$
\Pi_{x}=\exp \left\{-i \pi I_{x}\right\} .
$$

The positive sign in Eq. (A7) applies if the rotation angle differs from $\pi$ by an even multiple of $2 \pi$; the negative sign applies if the rotation angle differs from $\pi$ by an odd multiple of $2 \pi$. The propagators for the even-numbered elements of the $\mathrm{R} N_{n}^{v}$ sequence are therefore given by

$$
\begin{aligned}
U_{\mathrm{rf}}\left(\mathcal{E}_{\text {even }}\right) & = \pm \exp \left\{-i \Phi I_{z}\right\} \Pi_{x} \exp \left\{+i \Phi I_{z}\right\} \\
& = \pm \exp \left\{-2 i \Phi I_{z}\right\} \Pi_{x},
\end{aligned}
$$

where the phase shift $\Phi$ is given by

$$
\Phi=\frac{\pi \nu}{N}+\epsilon
$$

Here $\epsilon$ is an instrumental phase error. The rf fields for the odd and even elements of the $\mathrm{R} N_{n}^{\nu}$ sequence are related by a change in sign of the phase: This corresponds to a rotation of the rf field by $\pi$ around the $x$ axis. The propagator for the odd-numbered elements is therefore given by

$$
U_{\mathrm{rf}}\left(\mathcal{E}_{\text {odd }}\right)=\Pi_{x} U_{\mathrm{rf}}\left(\mathcal{E}_{\text {even }}\right) \Pi_{x}^{\dagger} .
$$

A pair of elements therefore generates the propagator

$$
U_{\mathrm{rf}}\left(\mathcal{E}_{\text {odd }}\right) U_{\mathrm{rf}}\left(\mathcal{E}_{\text {even }}\right)=\Pi_{x}^{2} \exp \left\{+4 i \Phi I_{z}\right\}=-\exp \left\{+4 i \Phi I_{z}\right\} .
$$

The complete sequence of $N / 2$ pairs generates the overall propagator

$$
U_{\mathrm{rf}}\left(\mathrm{R} N_{n}^{\nu}\right)=(-1)^{N / 2} \exp \left\{i 2 N \Phi I_{z}\right\} .
$$

This shows that the $\mathrm{R} N_{n}^{v}$ sequence rotates the spins by $-2 N \Phi$ about the $z$ axis. From Eq. (A10), this rotation angle is

$$
-2 N \Phi=-2 \pi \nu-2 N \epsilon,
$$

where $\epsilon$ is the misset in the rf phase shift, with respect to the ideal value. The overall $\mathrm{rf}$ propagator is given by

$$
U_{\mathrm{rf}}\left(\mathrm{R} N_{n}^{\nu}\right)=(-1)^{\nu+N / 2} \exp \left\{i 2 N \epsilon I_{z}\right\} .
$$

The overall sign of the rf propagator is usually of little consequence, but is included here for completeness.

\section{APPENDIX B: EFFECTIVE HAMILTONIANS OF TRANSFORMED CYCLES}

Consider two cycles, $A$ and $B$, for which the applied if interaction differs by a constant unitary transformation of the rf field at corresponding time points, i.e.,

$$
\mathcal{H}_{\mathrm{rf}}^{B}(t)=V(B, A) \mathcal{H}_{\mathrm{rf}}^{A}(t) V(A, B),
$$

where $V(A, B)=V(B, A)^{\dagger}$. The rf propagators for the two cycles are related by the same transformation,

$$
U_{\mathrm{rf}}^{B}\left(t_{b}, t_{a}\right)=V(B, A) U_{\mathrm{rf}}^{A}\left(t_{b}, t_{a}\right) V(A, B) .
$$

For example, the rf propagators for the cycles $\mathcal{S}_{0}$ and $\mathcal{S}_{\pi}$ are related by the operation $V\left(\mathcal{S}_{\pi}, \mathcal{S}_{0}\right)=\Pi_{z}$, while the the $\mathrm{rf}$ propagators for the cycles $\mathcal{S}_{0}$ and $\mathcal{S}_{0}^{\prime}$ are related by the operation $V\left(\mathcal{S}_{0}^{\prime}, \mathcal{S}_{0}\right)=\Pi_{x}$.

Now consider a particular internal spin interaction term $\mathcal{H}_{\Lambda}$, where $\Lambda$ refers to the dipole-dipole coupling, chemical shift, or any other spin interaction. In high field, the truncated form of this interaction term is

$$
\mathcal{H}_{\Lambda}(t)=\omega_{\Lambda}(t) T_{\lambda 0}^{\Lambda},
$$

where $T_{\lambda 0}^{\Lambda}$ is a component of an irreducible spherical tensor operator, and $\lambda$ is the spin rank of the interaction $\Lambda$. If $\mathrm{rf}$ sequence $A$ is applied, the spin interaction term may be transformed into the interaction frame of the rf field through

$$
\tilde{\mathcal{H}}_{\Lambda}^{A}(t)=U_{\mathrm{rf}}^{A}\left(t^{0}, t\right) \mathcal{H}_{\Lambda}(t) U_{\mathrm{rf}}^{A}\left(t, t^{0}\right),
$$

where $t^{0}$ is the starting point of the pulse sequence, and $U_{\mathrm{rf}}^{A}\left(t^{0}, t\right)=U_{\mathrm{rf}}^{A}\left(t, t^{0}\right)^{\dagger}$. Similarly, the spin interaction term for the sequence $B$ is given by

$$
\tilde{\mathcal{H}}_{\Lambda}^{B}(t)=U_{\mathrm{rf}}^{B}\left(t^{0}, t\right) \mathcal{H}_{\Lambda}(t) U_{\mathrm{rf}}^{B}\left(t, t^{0}\right),
$$

which may be written as 


$$
\begin{aligned}
\tilde{\mathcal{H}}_{\Lambda}^{B}(t) & =V(B, A) U_{\mathrm{rf}}^{A}\left(t^{0}, t\right) V(A, B) \mathcal{H}_{\Lambda}(t) V(B, A) U_{\mathrm{rf}}^{A}\left(t, t^{0}\right) V(A, B) \\
& =v_{\Lambda}(A, B) V(B, A) \tilde{\mathcal{H}}_{\Lambda}^{A}(t) V(A, B),
\end{aligned}
$$

where the number $v_{\Lambda}(A, B)$ is the parity of the spin tensor $T_{\lambda 0}^{\Lambda}$ under transformation by the operation $V(A, B)$, i.e.,

$$
V(A, B) T_{\lambda 0}^{\Lambda} V(B, A)=v_{\Lambda}(A, B) T_{\lambda 0}^{\Lambda} .
$$

For the cases of interest, the parity $v_{\Lambda}(A, B)$ is equal to \pm 1 , as will be discussed below.

The first-order average Hamiltonian for the interaction $\Lambda$ is given by the following integral:

$$
\overline{\mathcal{H}}_{\Lambda}^{(1)}\left(t, t^{0}\right)=\left(t-t^{0}\right)^{-1} \int_{t^{0}}^{t} d t^{\prime} \tilde{\mathcal{H}}_{\Lambda}\left(t^{\prime}\right)
$$

It follows from Eq. (84) that the first-order average Hamiltonians for the same interaction $\Lambda$ under the different cycles $A$ and $B$ are related through

$$
\overline{\mathcal{H}}_{\Lambda}^{(1)}(B)=v_{\Lambda}(A, B) \times V(B, A) \overline{\mathcal{H}}_{\Lambda}^{(1)}(A) V(A, B) .
$$

The second-order average Hamiltonian involving two interactions $\Lambda_{1}$ and $\Lambda_{2}$ is given by a double integral over a commutator,

$\overline{\mathcal{H}}_{\Lambda_{2} \Lambda_{1}}^{(2)}\left(t, t^{0}\right)=\frac{1}{2 i}\left(t-t^{0}\right)^{-1} \int_{t^{0}}^{t} d t^{\prime} \int_{t^{0}}^{t^{\prime}} d t^{\prime \prime}\left[\tilde{\mathcal{H}}_{\Lambda_{2}}\left(t^{\prime}\right), \tilde{\mathcal{H}}_{\Lambda_{1}}\left(t^{\prime \prime}\right)\right]$.

It follows that the second-order average Hamiltonians for the cycles $A$ and $B$ are related through

$\overline{\mathcal{H}}_{\Lambda_{2} \Lambda_{1}}^{(2)}(B)=v_{\Lambda_{2}}(A, B) v_{\Lambda_{1}}(A, B) V(B, A) \overline{\mathcal{H}}_{\Lambda_{2} \Lambda_{1}}^{(2)}(A) V(A, B)$.

In general, the $K$ th order average Hamiltonian terms are related for the two cycles by

$\overline{\mathcal{H}}_{\Lambda_{K} \cdot{ }^{\prime} \cdot \Lambda_{1}}^{(K)}(B)=\left(\prod_{Q=1}^{K} v_{\Lambda_{Q}}(A, B)\right) V(B, A) \overline{\mathcal{H}}_{\Lambda_{K} \cdots \Lambda_{1}}^{(K)}(A) V(A, B)$.

We may now use this formula to investigate the properties of some special cases.

\section{1. $\pi$-shifted cycles}

The rf fields of $\pi$-phase-shifted cycles are related by a $\pi$ rotation about the $z$ axis,

$$
V\left(\mathcal{S}_{\pi}, \mathcal{S}_{0}\right)=\Pi_{z},
$$

where $\Pi_{z}$ is given by

$$
\Pi_{z}=\exp \left\{-i \pi\left(I_{j z}+I_{k z}\right)\right\} .
$$

Since all high-field Hamiltonians are rotationally invariant about the $z$ axis, the parities of all interactions are given by

$$
v_{\Lambda_{Q}}\left(\mathcal{S}_{\pi}, \mathcal{S}_{0}\right)=1 \text {. }
$$

It follows from Eq. (B12) that all average Hamiltonian terms are related by

$$
\overline{\mathcal{H}}_{\Lambda_{K} \cdots \Lambda_{1}}^{(K)}\left(\mathcal{S}_{\pi}\right)=\Pi_{z} \overline{\mathcal{H}}_{\Lambda_{K} \cdots \Lambda_{1}}^{(K)}\left(\mathcal{S}_{0}\right) \Pi_{z}^{\dagger} .
$$

Hence all odd-quantum terms in the average Hamiltonian are inverted in sign when the rf pulse sequence is shifted in phase by $\pi$, while the even-quantum terms are unchanged. It follows that the recoupled double-quantum dipolar interaction, and the $z$ component of the chemical shift terms, are invariant to a $\pi$ phase shift of the recoupling sequence. The $x$ and $y$ components of the chemical shift terms change sign. These properties are included in Eq. (26).

\section{Phase-inverted cycles}

The transformations of the average Hamiltonian terms under phase inversion are less intuitive. The rf fields of phase-inverted cycles are related by a $\pi$ rotation about the $x$ axis,

$$
V\left(\mathcal{S}_{0}^{\prime}, \mathcal{S}_{0}\right)=\Pi_{x} .
$$

The parities of spin interactions under this transformation are given by

$$
v_{\Lambda_{Q}}\left(\mathcal{S}_{\pi}, \mathcal{S}_{0}\right)=(-1)^{\lambda} Q,
$$

where $\lambda_{Q}$ is the spin rank of interaction $\Lambda_{Q}$. The average Hamiltonian terms are therefore related by

$$
\overline{\mathcal{H}}_{\Lambda_{K} \cdots \Lambda_{1}}^{(K)}\left(\mathcal{S}_{0}^{\prime}\right)=(-1)^{\Sigma_{\lambda}} \times \Pi_{x} \overline{\mathcal{H}}_{\Lambda_{K} \cdots \Lambda_{1}}^{(K)}\left(\mathcal{S}_{0}\right) \Pi_{x}^{\dagger},
$$

where

$$
\Sigma_{\lambda}=\sum_{Q=1}^{K} \lambda_{Q}
$$

The effect of the phase inversion depends on whether the sum of the spin ranks of all the participating interactions is odd or even.

Consider the double-quantum dipole-dipole term, which appears in the first-order average Hamiltonian. Since the spin rank of this term is 2, the rank sum is also $\Sigma_{\lambda}=2$, and the first-order term for the phase-inverted cycle is given by

$$
\overline{\mathcal{H}}_{\mathrm{DQ}}\left(\mathcal{S}_{0}^{\prime}\right)=\Pi_{x} \overline{\mathcal{H}}_{\mathrm{DQ}}\left(\mathcal{S}_{0}\right) \Pi_{x}^{\dagger} .
$$

The double-quantum Hamiltonian is therefore rotated by $\pi$ about the $x$ axis for the phase-inverted cycle, as indicated in Eq. (31).

The second-order term is usually dominated by the chemical shift interactions. Since chemical shift interactions have spin rank $\lambda=1$, and two such interactions are involved in a second-order term, the rank sum is $\Sigma_{\lambda}=2$. As discussed in the text, the second-order chemical shift term is purely longitudinal in the case of $\mathrm{R} 26_{4}^{11}$, and dominates the term $\overline{\mathcal{H}}_{z}$. The longitudinal chemical shift term for the phaseinverted cycle is therefore given by

$$
\overline{\mathcal{H}}_{z}\left(\mathcal{S}_{0}^{\prime}\right) \cong \Pi_{x} \overline{\mathcal{H}}_{z}\left(\mathcal{S}_{0}\right) \Pi_{x}^{\dagger} .
$$

This implies that the second-order $\overline{\mathcal{H}}_{z}$ term changes sign upon phase inversion, 


$$
\overline{\mathcal{H}}_{z}\left(\mathcal{S}_{0}^{\prime}\right) \cong-\overline{\mathcal{H}}_{z}\left(\mathcal{S}_{0}\right)
$$

This important relationship accounts for the usefulness of the $\mathcal{S}_{0} \mathcal{S}_{0}^{\prime}$ supercycle in the context of symmetry-based recoupling.

The relationship in Eq. (B22) is only approximate since $\overline{\mathcal{H}}_{z}$ also contains a third-order term, which is invariant to phase inversion (see below).

The transverse chemical shift terms $\overline{\mathcal{H}}_{x}$ and $\overline{\mathcal{H}}_{y}$ appear in the third-order average Hamiltonian. Since the chemical shift terms have a spin rank of 1 , the third-order rank sum is $\Sigma_{\lambda}=3$, and the corresponding relationships are given by

$$
\begin{aligned}
& \overline{\mathcal{H}}_{x}\left(\mathcal{S}_{0}^{\prime}\right) \cong-\Pi_{x} \overline{\mathcal{H}}_{x}\left(\mathcal{S}_{0}\right) \Pi_{x}^{\dagger}, \\
& \overline{\mathcal{H}}_{x}\left(\mathcal{S}_{0}^{\prime}\right) \cong-\overline{\mathcal{H}}_{x}\left(\mathcal{S}_{0}\right),
\end{aligned}
$$

and

$$
\begin{aligned}
& \overline{\mathcal{H}}_{y}\left(\mathcal{S}_{0}^{\prime}\right) \cong-\Pi_{x} \overline{\mathcal{H}}_{y}\left(\mathcal{S}_{0}\right) \Pi_{x}^{\dagger}, \\
& \overline{\mathcal{H}}_{y}\left(\mathcal{S}_{0}^{\prime}\right) \cong+\overline{\mathcal{H}}_{y}\left(\mathcal{S}_{0}\right) .
\end{aligned}
$$

This implies that the $\overline{\mathcal{H}}_{x}$ term is changed in sign for the phase-inverted cycle, while the $\overline{\mathcal{H}}_{y}$ term is invariant to phase inversion. Similarly, the third-order contribution to the $\overline{\mathcal{H}}_{z}$ term is invariant to phase inversion.

${ }^{1}$ M. Mehring, High Resolution NMR in Solids (Springer, Berlin, 1982).

${ }^{2}$ U. Haeberlen, High Resolution NMR in Solids Selective Averaging (Academic, New York, 1976).

${ }^{3}$ K. Schmidt-Rohr and H. W. Spiess, Multidimensional Solid-State NMR and Polymers (Academic, London, 1994).

${ }^{4}$ M. J. Duer, Solid-State NMR. Principles and Applications (Blackwell Science, Oxford, 2002).

${ }^{5}$ S. R. Kiihne and H. J. M. de Groot, Perspectives on Solid State NMR in Biology (Kluwer, Dordrecht, 2001).

${ }^{6}$ M. Carravetta, X. Zhao, O. G. Johannessen et al., J. Am. Chem. Soc. 126, 3948 (2004).

${ }^{7}$ M. H. Levitt, D. P. Raleigh, F. Creuzet, and R. G. Griffin, J. Chem. Phys. 92, 6347 (1990).

${ }^{8}$ T. Gullion and J. Schaefer, J. Magn. Reson. (1969-1992) 81, 196 (1988).

${ }^{9}$ T. G. Oas, R. G. Griffin, and M. H. Levitt, J. Chem. Phys. 89, 692 (1988).

${ }^{10}$ M. H. Levitt, T. G. Oas, and R. G. Griffin, Isr. J. Chem. 28, 271 (1988).

${ }^{11}$ R. Tycko and G. Dabbagh, Chem. Phys. Lett. 173, 461 (1990).

${ }^{12}$ D. M. Gregory, M. A. Mehta, J. C. Shiels, and G. P. Drobny, J. Chem. Phys. 107, 28 (1997).

${ }^{13}$ M. A. Mehta, D. M. Gregory, S. Kiihne, D. J. Mitchell, M. E. Hatcher, J. C. Shiels, and G. P. Drobny, Solid State Nucl. Magn. Reson. 7, 211 (1996).

${ }^{14}$ S. Kiihne, M. A. Mehta, J. A. Stringer, D. M. Gregory, J. C. Shiels, and G. P. Drobny, J. Phys. Chem. A 102, 2274 (1998).

${ }^{15}$ A. E. Bennett, J. H. Ok, R. G. Griffin, and S. Vega, J. Chem. Phys. 96, 8624 (1992)

${ }^{16}$ Y. Ishii, J. Chem. Phys. 114, 8473 (2001).

${ }^{17}$ Y. Ishii, J. J. Balbach, and R. Tycko, Chem. Phys. 266, 231 (2001).

${ }^{18}$ N. C. Nielsen, H. Bildsøe, H. J. Jakobsen, and M. H. Levitt, J. Chem. Phys. 101, 1805 (1994)

${ }^{19}$ Y. K. Lee, N. D. Kurur, M. Helmle, O. G. Johannessen, N. C. Nielsen, and M. H. Levitt, Chem. Phys. Lett. 242, 304 (1995).

${ }^{20}$ M. Hohwy, H. J. Jakobsen, M. Edén, M. H. Levitt, and N. C. Nielsen, J. Chem. Phys. 108, 2686 (1998).

${ }^{21}$ A. Brinkmann, M. Edén, and M. H. Levitt, J. Chem. Phys. 112, 8539 (2000).

${ }^{22}$ M. Carravetta, M. Edén, A. Brinkmann, X. Zhao, and M. H. Levitt, Chem. Phys. Lett. 321, 205 (2000)
${ }^{23}$ M. Carravetta, M. Edén, O. G. Johannessen, H. Luthman, P. J. E. Verdegem, J. Lugtenburg, A. Sebald, and M. H. Levitt, J. Am. Chem. Soc. 123, $10628(2001)$

${ }^{24}$ P. E. Kristiansen, D. J. Mitchell, and J. N. S. Evans, J. Magn. Reson. 157, 253 (2002)

${ }^{25}$ P. E. Kristiansen, M. Carravetta, W. C. Lai, and M. H. Levitt, Chem. Phys. Lett. 390, 1 (2004)

${ }^{26}$ M. H. Levitt, in Encyclopedia of Nuclear Magnetic Resonance; Supplementary Volume, edited by D. M. Grant and R. K. Harris (Wiley, Chichester, UK, 2002).

${ }^{27}$ K. Schmidt-Rohr, Macromolecules 29, 3975 (1996).

${ }^{28}$ B. Reif, M. Henning, and C. Griesinger, Science 276, 1230 (1997).

${ }^{29}$ P. R. Costa, J. D. Gross, M. Hong, and R. G. Griffin, Chem. Phys. Lett. 280, 95 (1997).

${ }^{30}$ X. Feng, Y. K. Lee, D. Sandström, M. Edén, H. Maisel, A. Sebald, and M. H. Levitt, Chem. Phys. Lett. 257, 314 (1996).

${ }^{31}$ X. Feng, P. J. E. Verdegem, Y. K. Lee, D. Sandström, M. Edén, P. H. M. Bovee-Geurts, W. J. deGrip, J. Lugtenburg, H. J. M. de Groot, and M. H. Levitt, J. Am. Chem. Soc. 119, 6853 (1997).

${ }^{32}$ O. N. Antzutkin, J. J. Balbach, R. D. Leapman, N. W. Rizzo, J. Reed, and R. Tycko, Proc. Natl. Acad. Sci. U.S.A. 97, 13045 (2000).

${ }^{33}$ J. D. van Beek, L. Beaulieu, H. Schafer, M. Demura, T. Asakura, and B. H. Meier, Nature (London) 405, 1077 (2000).

${ }^{34}$ M. Hong, J. D. Gross, and R. G. Griffin, J. Phys. Chem. B 101, 5869 (1997).

${ }^{35}$ M. Hong, J. Magn. Reson. 139, 389 (1999).

${ }^{36}$ C. E. Hughes, Prog. Nucl. Magn. Reson. Spectrosc. 45, 301 (2004).

${ }^{37}$ J. Baum, M. Munowitz, A. N. Garroway, and A. Pines, J. Chem. Phys. 83, 2015 (1985).

${ }^{38}$ J. Baum and A. Pines, J. Am. Chem. Soc. 108, 7447 (1986).

${ }^{39}$ O. N. Antzutkin and R. Tycko, J. Chem. Phys. 110, 2749 (1999).

${ }^{40}$ H. Geen, R. Graf, A. S. D. Heinrichs, B. S. Hickman, I. Schnell, H. W. Spiess, and J. J. Titman, J. Magn. Reson. 138, 167 (1999).

${ }^{41}$ N. A. Oyler and R. Tycko, J. Phys. Chem. B 106, 8382 (2002).

${ }^{42}$ C. E. Hughes, J. Schmedt auf der Günne, and M. H. Levitt, ChemPhysChem 4, 457 (2003).

${ }^{43}$ T. Karlsson, J. M. Popham, J. R. Long, N. Oyler, and G. P. Drobny, J. Am. Chem. Soc. 125, 7394 (2003)

${ }^{44}$ S. Kiihne, K. B. Geahigan, N. A. Oyler, H. Zebroski, M. A. Mehta, and G. P. Drobny, J. Phys. Chem. A 103, 3890 (2003).

${ }^{45}$ D. H. Brouwer, R. J. Darton, R. E. Morris, and M. H. Levitt, J. Am. Chem. Soc. 127, 10365 (2004).

${ }^{46}$ D. H. Brouwer, P. E. Kristiansen, C. A. Fyfe, and M. H. Levitt, J. Am. Chem. Soc. 127, 542 (2005).

${ }^{47}$ U. Haeberlen and J. S. Waugh, Phys. Rev. 175, 453 (1968).

${ }^{48}$ A. Brinkmann and M. Edén, J. Chem. Phys. 120, 11726 (2004).

${ }^{49}$ A. Brinkmann and M. H. Levitt, J. Chem. Phys. 115, 357 (2001).

${ }^{50}$ M. Goldman, V. Fleury, and M. Guéron, J. Magn. Reson., Ser. A 118, 11 (1996).

${ }^{51}$ M. Edén, Chem. Phys. Lett. 366, 469 (2002).

${ }^{52}$ M. Edén and A. Brinkmann, J. Magn. Reson. 173, 259 (2005).

${ }^{53}$ A. D. Bain, J. Magn. Reson. (1969-1992) 56, 418 (1984).

${ }^{54}$ G. Bodenhausen, H. Kogler, and R. R. Ernst, J. Magn. Reson. (19691992) 58, 370 (1984).

${ }^{55}$ M. H. Levitt, P. K. Madhu, and C. E. Hughes, J. Magn. Reson. 155, 300 (2002).

${ }^{56}$ J. Jeener, Adv. Magn. Reson. 10, 1 (1982).

${ }^{57}$ K. T. Mueller, T. P. Jarvie, D. J. Aurentz, and B. W. Roberts, Chem. Phys. Lett. 242, 535 (1995).

${ }^{58}$ J. Schmedt auf der Günne, J. Magn. Reson. 165, 18 (2003).

${ }^{59}$ J. Schmedt auf der Günne, J. Beck, W. Hoffbauer, and P. Krieger-Beck, Chem.-Eur. J. 11, 4429 (2005).

${ }^{60} \mathrm{~J}$. Schmedt auf der Günne, J. Magn. Reson. 180, 186 (2006).

${ }^{61}$ M. Bak, J. T. Rasmussen, and N. C. Nielsen, J. Magn. Reson. 147, 296 (2000).

${ }^{62}$ H. Conroy, J. Chem. Phys. 47, 5307 (1967).

${ }^{63}$ S. K. Zaremba, Ann. Mat. Pura Appl. 4-54, 325 (1966).

${ }^{64}$ M. Groesbeek and J. Lugtenburg, Photochem. Photobiol. 56, 903 (1992).

${ }^{65}$ T. Hamanaka, T. Mitsui, T. Ashida, and M. Kakudo, Acta Crystallogr., Sect. B: Struct. Crystallogr. Cryst. Chem. 28, 214 (1972).

${ }^{66}$ H. Hosomi, Y. Ito, and S. Ohba, Acta Crystallogr., Sect. C: Cryst. Struct. Commun. 54, 142 (1998).

${ }^{67}$ P. G. Jönsson and A. Kvick, Acta Crystallogr., Sect. B: Struct. Crystallogr. Cryst. Chem. 28, 1827 (1972). 
${ }^{68}$ MATNMR is a toolbox for processing NMR/EPR data under Matlab, can be freely downloaded at http://matnmr.sourceforge.net

${ }^{69}$ A. E. Bennett, C. M. Rienstra, M. Auger, K. V. Lakshmi, and R. G. Griffin, J. Chem. Phys. 103, 6951 (1998).

${ }^{70}$ M. Bechmann, X. Helluy, and A. Sebald, J. Magn. Reson. 152, 14
(2001).

${ }^{71}$ J. D. van Beek, M. Carravetta, G. C. Antonioli, and M. H. Levitt, J. Chem. Phys. 122, 244510 (2005).

${ }^{72}$ T. Karlsson, A. Brinkmann, P. J. E. Verdegem, J. Lugtenburg, and M. H. Levitt, Solid State Nucl. Magn. Reson. 14, 43 (1999). 